\title{
Structural identification of neutralizing antibodies induced by nucleic acid encoded native-like trimer
}

\section{Ziyang Xu}

The Wistar Institute https://orcid.org/0000-0001-7690-7029

\section{Susanne Walker}

The Wistar Institute

\section{Megan Wise}

Inovio Pharmaceuticals

Neethu Chokkalingam

The Wistar Institute

\section{Mansi Purwar}

The Wistar Institute

\section{Alan Moore \\ Indiana University}

\section{Edgar Tello-Ruiz}

The Wistar Institute

\section{Yuanhan Wu}

The Wistar Institute https://orcid.org/0000-0002-4706-439X

\section{Sonali Majumdar}

Wistar Institute

\section{Faraz Zaidi}

The Wistar Institute

\section{Emma Reuschel}

The Wistar Institute https://orcid.org/0000-0002-4627-1354

\section{Ishaan Patel}

The Wistar Institute

\section{April Obeirne}

The Wistar Institute

Jianqiu Du

Indiana University

Katherine Schultheis

Inovio Pharmaceuticals

\section{Lauren Gites}

Inovio Pharmaceuticals

Trevor Smith 
Inovio Pharmaceuticals

Janess Mendoza

Inovio Pharmaceuticals

Kate Broderick

Inovio Pharmaceuticals (United States)

Laurent Humeau

Inovio Pharmaceuticals

Jesper Pallesen

The Scripps Research Institute https://orcid.org/0000-0002-3270-1587

David Weiner

The Wistar Institute https://orcid.org/0000-0002-2232-8512

Daniel Kulp ( $\sim$ dwkulp@wistar.org )

Wistar Institute https://orcid.org/0000-0002-0527-3804

\section{Article}

Keywords: HIV Vaccine, HIV Envelope, Autologous Neutralizing Antibodies, Informed Humoral Response, In Vivo Folding, Structured Antigens

Posted Date: March 15th, 2021

DOl: https://doi.org/10.21203/rs.3.rs-275402/v1

License: (c) (i) This work is licensed under a Creative Commons Attribution 4.0 International License. Read Full License

Version of Record: A version of this preprint was published at Nature Communications on February 4th, 2022. See the published version at https://doi.org/10.1038/s41467-022-28363-z. 


\section{Structural identification of neutralizing antibodies induced by} nucleic acid encoded native-like trimer

Ziyang Xu ${ }^{1,2 \#}$, Susanne Walker ${ }^{1 \#}$, Megan C. Wise ${ }^{3 \#}$, Neethu Chokkalingam ${ }^{1 \#}$, Mansi Purwar ${ }^{1 \#}$, Alan Moore ${ }^{4}$, Edgar Tello-Ruiz ${ }^{1}$, Yuanhan $\mathrm{Wu}^{1}$, Sonali Majumdar ${ }^{1}$, Faraz I. Zaidi $^{1}$, Emma L. Reuschel ${ }^{1}$, Ishaan Patel ${ }^{1}$, April Obeirne ${ }^{1}$, Jianqiu $\mathrm{Du}^{4}$, Katherine Schultheis ${ }^{3}$, Lauren $\mathrm{Gites}^{3}$, Trevor Smith ${ }^{3}$, Janess Mendoza ${ }^{3}$, Kate E. Broderick ${ }^{3}$, Laurent Humeau ${ }^{3}$, Jesper Pallesen ${ }^{4}$, David B. Weiner ${ }^{1}$, Daniel W. Kulp ${ }^{1 *}$

${ }^{1}$ Vaccine and Immunotherapy Center, The Wistar Institute, Philadelphia, PA 19104

2 Perelman School of Medicine, University of Pennsylvania, Philadelphia, PA 19104.

${ }^{3}$ Inovio Pharmaceuticals, Plymouth Meeting, PA, 19462

${ }^{4}$ Molecular and Cellular Biochemistry, Indiana University, Bloomington, IN, 47405

*Corresponding Author: dwkulp@wistar.org

\#These authors contributed equally to this work

\section{Abstract}

Despite extensive efforts, an efficacious HIV vaccine remains elusive. HIV Envelope (Env) is the target of all known neutralizing antibodies. Stabilizing Env into native-like trimer (NLT) conformations is required for recombinant protein immunogens to induce autologous neutralizing antibodies(nAbs) against difficult to neutralize HIV strains (tier-2) in rabbits and non-human primates. However, mice are the most cost effective and widely used vaccine model and mouse immunizations with NLTs have generally failed to induce tier-2 nAbs. Here, we explored if DNA-encoded NLTs would fold properly in vivo and induce an informed humoral response. We observed DNA encoding a BG505 NLT induces autologous tier-2 nAbs in mice, identified murine monoclonal neutralizing antibodies, mapped the responses to a new murine neutralizing Env C3/V5 epitope and determined the structures of Env-nAbs complexes. Beyond potential functional immunity gains, DNA vaccines permit in vivo folding of structured antigens and provide significant cost and speed advantages for enabling rapid evaluation of new HIV vaccines.

\section{Introduction}

The diversity and unique glycosylation profiles of the HIV-1 surface protein, Envelope (Env), hamper development of an efficacious prophylactic HIV-1 vaccine that induces broadly neutralizing antibody (bNAb) responses ${ }^{1,2}$. The early generations of HIV-1 vaccines included the monomeric gp120 domain or non-native gp140/160 forms of Env. Though these vaccines induced binding antibodies which 
recognized Env, the responses were not able to neutralize circulating HIV-1 viruses ${ }^{3,4}$. Over the years, various modifications to the Env protein were explored to create trimeric proteins. Early iterations included the use of foldon or isoleucine zipper domains to form non-native trimer structures ${ }^{5,6}$. In recent years, several modifications have been found to allow the folding and production of trimer proteins that resemble the native viral Env conformations, or native-like trimers (NLTs) ${ }^{7-11}$. Biophysical technique such as cryo-electron microscopy (cryo-EM) and antigenic profile characterization by X-ray crystallography of various identified broadly neutralizing antibodies (bNAbs) bound to NLTs confirmed that these NLTs can present important conformational neutralizing epitopes in a solvent-accessible fashion ${ }^{8,12}$. Importantly, when rabbits and non-human primates (NHPs) were immunized with adjuvant co-formulated protein NLTs, autologous tier-2 neutralizing antibodies were induced and the responses from a select group of vaccinated animals were able to protect some NHPs from an autologous tier-2 SHIV challenge ${ }^{13-15}$.

However, experiments using rabbits and macaques are costly and lack throughput. For many infectious diseases, mice have been identified as an ideal small laboratory animal for preliminary screening and down-selection of designed immunogens, prior to evaluations of the promising clinical candidates in larger animals ${ }^{16}$. In the case of HIV-1, however, it has been demonstrated that induction of autologous Tier 2 neutralizing antibody responses with protein NLTs in mice were extremely difficult, regardless of the choice of mouse strains, adjuvants, or routes of vaccination (subcutaneous depot or slow-release mechanism using an osmotic pump $)^{17,18}$. Several hypotheses were proposed for these findings, namely the immune-dominance of epitopes at the base of soluble NLTs which are not normally exposed in native virions, as well as deficiencies in mouse antibody repertoire to recognize glycancontaining epitopes ${ }^{8,17}$. Many subsequent studies with protein NLTs were, therefore, not performed in mice and advanced directly for evaluations in rabbits and macaques ${ }^{19-21}$, even though it has been shown in a more recent study that scaffolding of NLTs in a multi-valent fashion on nanoparticle scaffolds may facilitate the induction of autologous Tier 2 antibody responses in mice 22 .

DNA vaccines are an approach where engineered nucleic acids are administered to a host for in vivo production of an antigen to elicit immune responses ${ }^{23}$. While early studies demonstrated the immunogenicity of DNA vaccines in larger animals and humans was sub-optimal, advances in antigen design, nucleic acid formulations, genetic adjuvants and adaptive electroporation (EP) technologies have now enabled DNA vaccines to induce more potent and consistent responses in several clinical studies, including Zika, MERS and Ebola ${ }^{24-26}$. In the HIV space, a DNA vaccine encoding two consensus Envs, Gag, and Pol antigens adjuvanted with plasmid IL-12 delivered by intradermal electroporation induced $98 \%$ sero-conversion, tier 1 neutralization and strong cellular responses in the HVTN098 study ${ }^{27}$. Importantly, 
we have also demonstrated that DNA/EP can be used for direct in vivo delivery of highly-folded monoclonal antibodies and biologics ${ }^{28-30}$, and to launch de novo assembly of more complex nanoparticle vaccines in the hosts to induce immune responses phenotypically different from protein nanoparticle vaccines $^{31}$.

In this work, we attempted to determine if DNA/EP can be used for in vivo expression of a conformationally complex NLT and, if so, characterize immune responses induced by DNA versus protein NLT vaccinations using previously well-characterized stabilized NLT BG505.MD39 as a model antigen ${ }^{32}$. We observed DNA/EP resulted in direct in vivo production and proper assembly of NLTs using blue NATIVE PAGE analysis as well as staining of tissues with bNAbs and non-nAbs. Comparison of protein versus DNA MD39 vaccinations in mice demonstrated induction of trimer-binding antibodies by both routes. However, CD4+ and CD8+ T-cell responses, as well as autologous Tier 2 BG505.T332N neutralization was observed in mice immunized with DNA but not protein MD39. T-cell and neutralizing antibody (nAb) responses induced by DNA NLT were found to be further improved by increasing the interval between vaccinations. We further characterized the nAb by engineering pseudo-virus variants with modified glycosylation sites and single cell RNA-sequencing on sorted antigen-specific B-cells. Five unique murine neutralizing antibody clones were isolated and three were characterized with cryo-EM and found to be specific for a neutralizing epitope on the V5 loop of Env trimer. This study demonstrated that DNA/EP can be used for direct in vivo expression of conformationally complex antigens such as NLT and can elicit phenotypically different immune responses, particularly $n A b$ responses, consistently in animals. Further studies to compare these two routes of immunizations and to explore the unique properties of DNA vaccines such as inducing T-cell and nAb responses are likely important for HIV-1 and other infectious diseases.

\section{$\underline{\text { Results }}$}

Synthetic DNA (synDNA) encoded native-like trimers properly fold, express and maintain antigenic profile in vitro and in vivo

We optimized in vivo expression of BG505.MD39 by performing codon and RNA optimization of the antigen nucleotide sequence and including an N-terminal IgE leader sequence which facilitated both antigen trafficking to the secretory pathway and ribosomal loading ${ }^{28,33}$. To ensure intact biophysical and antigenic profiles for the optimized BG505.MD39, we expressed the construct in vitro using Expi293F cells. Size Exclusion Chromatography demonstrated homogeneous assembly of lectin purified BG505.MD39 which eluted as a single fraction that corresponds to a trimer (Figure 1A). Using binding ELISA, expressed BG505.MD39 was shown to bind to bNAb PGT145, which preferentially recognizes the trimeric 
conformational epitope on Env V2 apex ${ }^{34}$, but not non-NAb 3074, which is specific for the Env V3 loop which is an epitope exposed on monomeric gp140 but hidden in a NLT ${ }^{35-37}$ (Figure 1B). A full antigenic profile of the optimized BG505.MD39 is consistent with previous studies (Supplemental figure S1A-C) ${ }^{8,32}$. Negative-stain Electron Microscopy (nsEM) demonstrated intact trimeric propeller-shaped conformation of BG505.MD3915 (Figure 1C).

We characterized the biophysical and antigenic profiles of DNA-delivered in vivo produced NLTs. To our knowledge, this type of in vivo profiling of NLTs has not been previously studied. Muscle homogenates of BALB/c mice intramuscularly inoculated with plasmid BG505.MD39 (pMD39_opt) were obtained four days post injection (d.p.i). We first visualized how purified BG505.MD39 and recombinant BG505.gp120 proteins would migrate on a blue NATIVE PAGE gel using Coomassie staining (Figure 1D). While analysis of glycoproteins with PAGE is challenging, we observed BG505.MD39 standard migrated as a trimer at a molecular weight (MW) of $\sim 480 \mathrm{kDa}$. In comparison, BG505.gp120, as expected, migrated mainly as a monomer, and a small fraction as dimer, on the NATIVE PAGE gel at MWs of 120 and 240kDa, respectively. As muscle homogenates contain proteins other than BG505.MD39, we used 2G12based Western analysis to more specifically detect bands that correspond to HIV Env proteins. We observed that BG505.MD39 in muscle homogenates migrated mainly as trimers in blue NATIVE PAGE gel in comparison to the protein BG505.MD39 standard, with small fractions existing as monomers or dimers as is consistent with domain-wise global folding pathways of newly synthesized polypeptides ${ }^{38}$ (Figure 1E). of in vivo versus in vitro produced BG505.MD39 may reflect differences in glycosylation profiles between Exp293F cell line versus murine muscle cell produced glycoproteins. A critical characteristic of a vaccine immunogen is retention of proper conformation in vivo. We developed an experiment to assess in vivo antigenic profiling by immune-fluorescence (IVAP). Briefly, BALB/c mice are injected with DNA plasmids and muscles are harvested seven d.p.i and stained with bnAbs or non-nAbs, followed by fluorophoreconjugated anti-human secondary antibody. We conducted an IVAP experiment with empty backbone (pVAX1), pMD39_opt, or pGP120_foldon (a non-native like trimer of gp120s) (Figure 1F). Muscle injected with pMD39_opt had strong staining for trimer-specific bnAbs (PGT145) but not a V3-directed non-nAbs (3074). In contrast, muscles injected with pGP120_foldon had strong staining with 3074 and not PGT145. The IVAP analysis demonstrated DNA-delivered NLTs express well-folded trimeric immunogens, supporting the use of DNA/EP for delivery of conformationally complex HIV immunogens.

DNA encoded NLT immunization induced stronger T-cell and nAb responses as compared to corresponding protein immunization in mice 
We next compared immune responses induced by protein and DNA-encoded BG505.MD39 in mice. BALB/c mice were immunized with the same dose (25ug) of either DNA-encoded or RIBI formulated protein BG505.MD39 delivered at weeks 0, 3, and 6. Two weeks after final immunization, mice were sacrificed, and cellular responses were determined using 15 mer overlapping peptides that span the wildtype (WT) BG505 Envelope. As measured by the IFNY ELISpot assay, mice vaccinated with pMD39_opt developed significantly stronger Env-specific T-cell responses than mice immunized with protein BG505.MD39 (Figure 2A). These antigen specific cellular responses generated recall from peptides spanning multiple regions of Env. Importantly, both $\mathrm{CD} 4^{+}$and $\mathrm{CD} 8^{+} \mathrm{T}$ cell responses were induced by DNA vaccination (Figure 2B), and antigen-specific $\mathrm{CD}^{+}$and $\mathrm{CD} 8^{+} \mathrm{T}$ cells exhibited significant poly-functionality in terms of simultaneous expression of IFN- $\gamma$, TNF $\alpha$, and IL-2 (Supplemental figures S2, S3A-B). Activation of both T follicular helper (Tfh) cells and germinal center (GC) B cells has been demonstrated to be instrumental to the induction of humoral responses ${ }^{39,40}$. Here, we characterized the kinetics of Tfh and GC B cell responses in muscle draining lymph nodes and spleen following EP mediated pMD39_opt delivery. It was observed that frequency of $\mathrm{CD} 4^{+} \mathrm{CD} 44^{+} \mathrm{PD} 1^{+} \mathrm{CXCR} 5^{+} \mathrm{Tfh}$ cells peaked around 7 d.p.i whereas frequency of $\mathrm{CD} 19^{+} \mathrm{GL7} 7^{+} \mathrm{GC}$ B cells increased past 10 d.p.i in the draining lymph nodes ${ }^{41}$ (Supplemental figure S4A-B). However, we did not observe increased frequency in either population in the spleen. Next, we compared Tfh and GC B cell responses mice immunized with either DNA-encoded or RIBI-coformulated protein BG505.MD39 in the draining lymph nodes 10 d.p.i, and observed increased frequencies in Tfh and GC B cells in mice immunized with DNA versus those immunized with protein (Figure 2C-D). In addition, higher frequencies of Tfh cells were induced by DNA BG505 NLT than compared to previously published Tfh frequencies for BG505.SOSIP immunizations in mice ${ }^{17}$.

We next characterized humoral responses induced in mice following DNA-encoded versus protein BG505.MD39 vaccination. While DNA-encoded BG505.MD39 induced stronger trimer (BG505.MD39)specific binding antibody responses than protein BG505.MD39 at week 2 and week 5, binding titers at week 8 were similar (Figure 2E). Strikingly, however, autologous Tier 2 neutralization against BG505.T332N using the TZM-bl assay were uniquely detected in 3 out of 10 mice immunized with DNAencoded BG505.MD39, but not in naïve mice, mice immunized with pVAX1 plasmid backbone, or those immunized with protein BG505.MD39 two weeks post the third vaccination (Figure 2F and Supplemental Table S1). Non-specific neutralization of MLV was not observed in any of the post-immune sera in our assays.

\section{nAb responses increased with lengthened vaccination intervals}


While we observed a striking phenotypic difference between DNA versus protein BG505.MD39 immunizations in terms of induction of nAbs, few responders in the study successfully developed $n A b$ responses, and the $\mathrm{ID}_{50}$ titers (sera dilution that corresponds to $50 \%$ reduction in infection by pseudovirus) induced in the responders were low. Longer intervals between vaccinations have previously been demonstrated to induce superior antibody responses by allowing time for somatic hypermutation and affinity maturation ${ }^{42,43}$. We next explored if lengthening the interval between the second and third immunizations could increase the frequency of responders and magnitudes of responses.

BALB/c mice were immunized with 25ug of DNA-encoded MD39 at weeks $0,3,6$ or weeks 0,3 , 16 (Figure 3A). Mice immunized with the longer interval induced significantly higher antigen specific T cell responses as measured by IFN- $\gamma$ ELIspot assays compared to mice receiving the shorter immunization schedule (Figure 3B). In both cases, antigen-specific $\mathrm{CD}^{+}$and $\mathrm{CD} 8^{+} \mathrm{T}$-cells were induced by DNA vaccinations (Figure 3C). Interestingly, vaccination using the long interval scheme induced stronger

172 polyfunctional $\mathrm{CD}^{+} \mathrm{T}$-cell responses whereas vaccination with the short interval scheme induced stronger 173 polyfunctional $\mathrm{CD}^{+} \mathrm{T}$-cell responses as measured two weeks post last boost (Supplemental figure S5A174 B). Both vaccine regimens induced strong trimer-specific binding antibodies with comparable titers two weeks post the final vaccination (Figure 3D-F). It was observed that antibody responses did not contract significantly from week 5 (post $2^{\text {nd }}$ immunization) to week 16 (prior to $3^{\text {rd }}$ immunization) (Figure 3E).

177 Importantly, in terms of nAb responses, it was observed that there was a higher frequency of responders

178 (7 out of 10 mice) that developed autologous Tier-2 nAbs for the long vaccination scheme as compared 179 to the short vaccination scheme (3 out of 10 mice) (Figure 3G). nAb ID 50 titers in the responders were also 180 found to be improved for mice which received the long immunization scheme (Supplemental Table S2). 181 It may therefore be concluded that the potency and consistency of nAb responses induced by DNA182 encoded NLTs may be further improved with optimized immunization regimens.

Screening with engineered pseudoviruses mapped induced murine nAb responses to the Env C3/V5 epitope

We further interrogated nAbs induced in mice by determining the neutralization sensitivity to engineered pseudoviruses. BG505.T332N pseudovirus was used for mapping studies as the vaccine encoded stabilized BG505.MD39 also contains this corresponding glycan addition. Soluble BG505.SOSIP.664 protein could induce autologous Tier 2 neutralizing antibodies in rabbits, which were specific for the S241 glycan hole ${ }^{44}$ (Figure 4A), and the V5 loop (C3/V5 epitope) in the case of rabbits and 
induced murine nAbs might be specific for these previously identified neutralizing epitopes, we engineered and titered two additional pseudoviruses containing point mutations BG505.T332N.S241N or BG505.T332N.T465N with a rationale that the introduced $\mathrm{N}$-linked glycan may block binding and neutralization mediated by epitope-specific antibodies ${ }^{48}$. We performed screening using post-immune mice sera that have previously been demonstrated to possess potent neutralizing activities against the autologous BG505.T332N virus. We observed that while the murine nAbs were capable of neutralizing the S241N isolate almost as effectively, nAbs were far less potent in their activities against the T465N isolate, suggesting that the induced nAbs likely target the V5 loop but not the S241 glycan hole (Figure 4C). Expectedly, induced murine nAbs also failed to neutralize the maternal MG505.H3 isolate, which differed in its sequence of the V5 loop by two residues ( $T$ to $N$ changes) as compared to the BG505 isolate $\mathrm{e}^{19,49}$ (Figure 4D). We did not observe neutralization of any of the pseudovirus isolates in the global panel ${ }^{50}$.

\section{Single Cell RNA sequencing on antigen-specific B-cell recovered monoclonal murine nAb that targeted} the V5 loop

To further validate our findings and characterize induced murine nAb responses, we attempted to recover monoclonal antibodies from an immunized animal (272.8) that developed nAb responses against the BG505.T332N isolate following DNA-encoded BG505.MD39 immunization. The animal was immunized according to the long vaccination regimen (Wks $0,3,16)$ (Figure 5A), and was euthanized two weeks post the final vaccination for tissue (spleen and draining lymph nodes) collection. At the final timepoint, potent trimer-binding antibodies were induced in 272.8 (Figure 5B). Serum of the animal also demonstrated strong neutralization activity against the BG505.T332N isolate, conferring almost complete neutralization of the virus at a low dilution (Figure 5C). Collected spleen and lymph nodes were homogenized into a single cell suspension and stained with relevant surface antibodies and fluorophoreconjugated streptavidin-scaffolded avi-tagged BG505.MD39 tetramers as previously described ${ }^{8,51}$. Viable antigen-specific CD19+ IgM-IgD- MD39_Tetramer_FITC ${ }^{+}$MD39_Tetramer_PE ${ }^{+}$B cells were subsequently sorted out in bulk with a FACS ARIA II instrument (Figure 5D) for single-cell RNA sequencing. By using the 10x genomics platform, it was possible to retrieve paired heavy and light chain sequences for each $B$ cell that had been bulk sorted ${ }^{52}$. Following cDNA prep, enrichment of VH and VL DNA fragments, and nextgeneration high-throughput sequencing, 25 unique clonotypes were identified (Supplemental figure S6AB). 18 unique germline $\mathrm{VH}$ genes were identified among the 25 clonotypes as well as 16 unique germline VK genes with a high frequency of one IGHV/IGKV pairing (IGHV6-6/IGKV3-2) (Figure 5E and Table 1). While the barcode frequency of the IGHV6-6/IGKV3-2 antibody pairs was numerous, the highest barcode 
frequency of a single clonotype was from an IGHV3-2/IGKV4-91 antibody pair, clone 3 (C03) (Table 1). The 25 unique clonotypes displayed variable HCDR3 lengths from 6 amino acids to 17 amino acids and variable HCDR3 sequences, however the 6 common IGHV6-6 clonotypes were consistent in both HCDR3 length and had HCDR3 sequence convergence (Supplemental figure S6B).

Next, we generated a 2 plasmid-system DNA assembly of the enriched clonotypes. One plasmid consisting of each variable heavy fragment upstream of human IgG1 Fc/CH1 and the other plasmid containing each variable light fragment upstream of the constant domain of human Kappa light chain. These were dually expressed using small-scale transfections of Expi293F cells and the supernatants screened with MD39 trimer-binding ELISA (Figure 6A). 5 out of 25 clones (C05, C22, C24, C33, and C37) demonstrated appreciable binding to both MD39 and gp120-foldon with similar affinities in terms of EC50 (Supplemental figure S7A-C) and their in vitro expression was characterized by western blot (Supplemental figure S7D). Antibody concentrations of the supernatants were calculated by standard ELISA binding curves for the 5 clones using purified IgG (Supplemental figure S7E). We next evaluated the neutralizing activities of the selected clones. All of the clones demonstrated appreciable neutralizing activities against BG505.T332N to varying degrees (Figure 6B), the potency of which were observed to be similar to rhesus monoclonal antibodies $1 \mathrm{~B} 1$ and $1 \mathrm{~B} 6\left(\mathrm{IC}_{50} \text { values of } 0.03 \text { and } 0.06 \mathrm{ug} / \mathrm{mL} \text { respectively }\right)^{47}$. Simultaneously, none of the clones could completely neutralize BG505.T332N.T465N (Figure 6C), which remained sensitive to neutralization by V3 N332-directed bNAb PGT128 (Supplemental figure S7F) ${ }^{53}$, demonstrating V5-specificities of the isolated clones. In comparison, PGT128 neutralized BG505.T332N.T465N as efficiently as it did for BG505.T332N (Supplemental figure S7F). One clone, C24, demonstrated modest neutralization of the BG505.T332N.T465N which could indicate a slightly modified binding epitope from the other clones. None of the isolated murine mAb clones non-specifically neutralized control pseudovirus MLV (Figure 6D). Overall, the 5 isolated clones displayed different potencies in the neutralization of autologous BG505.T332N pseudovirus, with C05 and C37 being the most potent clones, with $\mathrm{IC}_{50}$ values of $37 \mathrm{ng} / \mathrm{mL}$ and $47 \mathrm{ng} / \mathrm{mL}$ respectively (Figure $6 \mathrm{E}$ ). The 5 functional clones were of the same common antibody lineage IGHV6-6/IGKV3-2 with similar HCDR3 loops (Figure 6F). The kappa light chains showed few variances between clonotypes ranging from 2 amino acids to 11 amino acids per variable gene. The heavy chains displayed more variation with 7 amino acids to 15 amino acids per gene (Supplemental figure S8A). Clonotypes C05, C24 and C33 were structurally modeled and compared due to the differences in $\mathrm{IC}_{50}$ values and neutralization of BG505.T332N.T465N pseudovirus. A previously crystalized murine IGHV6-6/IGKV3-2 paired antibody was used as a template ${ }^{54}$ and the somatic mutations and variable loops were modeled with Rosetta (Figure 6G). Some mutations were common 
among multiple clonotypes (Figure 6G, red spheres) such as the N35S mutation found on the HCDR1 loop which was mutated in four out of the five functional antibodies while many framework mutations were unique to each clone (Figure 6G, grey spheres). Clone C33 had the fewest mutations away from the germline (only 3 mutations in total) and the weakest neutralization against BG505.T332N pseudovirus. C24 has a greater number of CDR loop mutations compared to C05 which could account for the differences in neutralization of the BG505.T332N.T465N pseudovirus (Supplemental figure S8B). To further investigate epitope specificity of C24 antibody in comparison with other isolated antibodies, we performed competition ELISA and determined whether each of the following non-biotinylated antibodies (C05, C22, C24, C33, C37 and PGT128) could block the binding of biotinylated C24 to MD39 (Supplemental figure S9A). We observed that while C05, C22, and C37 could outcompete the binding of biotinylated C24 to MD39, C33 and PGT128 were unable to do so (Supplemental figure S9A-B), suggesting while C05, C22, C24 and C37 may bind to MD39 in a similar fashion, C33 may approach MD39 from a slightly different angle.

Cryo-EM characterization of Fab of murine nAb bound to MD39 provided a structural basis for C3/V5directed neutralization

To confirm our findings that the C3/V5 epitope is the precise target of the monoclonal nAbs, we employed Cryo-EM electron microscopy. Three nAbs were selected for structural characterization: C24 which retained some neutralization to BG505.T465N pseudovirus (Figure 6E), C05 which is the most potent neutralizer of BG505 pseudovirus (Figure 6E), and C33 which is the least mutated of the isolated antibodies (Figure S7B). We recorded 1517 movie micrographs and obtained a 7.4A resolution structure from 5971 molecular projection images of BG505.MD39 in complex with C24 (Figure 7A). Alignment of Env structure (PDB ID: 6VOR) allowed us to visualize the epitope at the interface with the C24 Fab, confirming interactions with the C3/V5 epitope (Figure 7B). We took 1,283 micrographs and obtained a 7Å resolution from 37,073 molecular projection images of BG505.MD39 in complex with C05 (Figure 7C). We took 2,080 micrographs and obtained a $4.8 \AA ̊$ resolution structure from 70,242 projection images of BG505.MD39 in complex with C33 (Figure 7C).

Next, we sought to compare structures of the murine C3/V5 neutralizing antibodies to structures of C3/V5 targeting antibodies from other species. The C3/V5 epitope has been primarily mapped by polyclonal electron microscopy and has been identified in NLT-immunized rabbits ${ }^{55}$, NLT-immunized macaques $^{13,45-47}$ and SHIV-infected macaques ${ }^{45,47}$. The antibodies from these studies engage the C3/V5 
epitope from various angles of approach. The murine antibodies seem to engage the epitope most similarly to antibodies in a polyclonal EM structure from a NLT vaccinated rhesus macaque with high titers of neutralizing antibodies, which was fully protected after 12 SHIV challenges ${ }^{45}$ (Figure 7D). While the polyclonal EM method is incredibly powerful, it does not map functionality of each clone to the structure. Here, we have mapping of C3/V5-targeting antibody structure and function, allowing us to identify the C3/V5 engagement geometry that is consistent with neutralization.

In summary, using current molecular biology and genomics techniques, we identified 5 unique and novel murine C3/V5-directed nAbs and validated that mice can mount epitope-specific nAb against an autologous Tier 2 isolate following DNA-encoded NLT immunization.

\section{$\underline{\text { Discussion }}$}

While iterative vaccine studies are critical to the development of an efficacious HIV vaccine, a significant bottleneck for studying NLT-induced neutralizing immune responses has been the reliance on large animal models. To compound the problem of increased cost and lengthy experiment times associated with larger animals, recombinant protein immunogens required in most NLT studies to date necessitates costly and arduous expression and purification approaches. Here, we present a potential breakthrough in this long-standing bottleneck in HIV research and demonstrate a simple, rapid and costeffective strategy to screen for antigens that elicit neutralizing antibodies, which involves immunization of BALB/c mice with DNA-encoded NLTs to enable rapid immunological read-outs.

A long-time collective effort in HIV vaccine research has been focused on producing immunogens with proper trimer folding and concomitant neutralizing antibody responses. DNA NLTs are produced in vivo and so it was therefore important to confirm assembly of our NLT immunogen in vivo. Previous studies involving DNA vaccination of NLTs did not directly measure antigen assembly in the animals and did not demonstrate that DNA vaccinations alone could induce Tier 2 autologous neutralizing antibodies ${ }^{36}$. In this study, we sought to provide a more direct assessment of in vivo assembly of conformationally complex HIV NLT antigens. First, we performed Native PAGE electrophoresis on immunized muscle homogenates to obtain protein separation of material being produced in vivo roughly by molecular weight. This demonstrated that we have bands which migrate similarly to the recombinantly produced material. The migration is slightly different, likely due to glycosylation differences by the host cell type, which will require further study. Second, we performed in vivo antigenic profiling of NLTs for what we 
believe is the first time. We observed that DNA/EP delivery of BG505.MD39 results in direct in vivo production of trimeric antigens, which retain important conformational epitopes (e.g. V2-apex site) that is unique to NLT Env immunogens.

The neutralizing specificities elicited from the murine antibody repertoire against NLTs relative to other animal models has not been well described. The serological and structural data generated by BG505.MD39 immunizations shows for the first time that immune systems of BALB/c mice can target the C3/V5 neutralizing epitope which is a broadly targeted epitope capable of mediating protection from viral challenges in other large animal species ${ }^{13,15,20,45-47}$. Interestingly, in a recent bolus vs extended dose study of BG505 NLTs in rhesus macaques, the extended dose group uniquely targeted C3/V5 and these animals had much higher neutralizing titers ${ }^{46}$. In electron microscopy-based mapping studies using polyclonal sera the C3/V5 epitope can be engaged from a number of different angles of approach ${ }^{45}$. Here, we use this in vivo NLT immunization model to generate a collection of mAb that allow us to define the angle of approach that is used to neutralize via the C3/V5 epitope by determining a cryo-EM structure of bound monoclonal neutralizing abs. This cross-species epitope may be a relevant target of vaccine-induced human immunity.

By comparing protein to DNA immunizations of BG505.MD39 in mice at the equivalent dose, we observed different immunity. This was unexcepted as both routes ultimately should be presenting the same structure and distinct epitopes to the immune system. While both protein and DNA vaccination induced trimer-specific binding antibodies, DNA but not protein vaccination induced $\mathrm{CD} 4^{+}$and $\mathrm{CD} 8^{+} \mathrm{T}$-cell responses as well as nAb responses. Differences in the mechanisms with which antigens traffic and present for protein versus DNA-encoded immunogens may underlie differential induction of immune phenotypes by these two routes of vaccination ${ }^{56}$. Protein antigens are supplied as a depot of soluble antigens and may rely on bulk flow in the lymphatic system for antigen trafficking ${ }^{57}$. In contrast, it has 343 been shown that DNA vaccination may either result in direct transfection of antigen-presenting cells (APCs) to express the encoded antigen or may result in transfection of myocytes which can then transfer the expressed antigens to infiltrating APCs through the formation of apoptotic bodies ${ }^{58,59}$. Such differences in antigen-presentation may partially explain the unique induction of $\mathrm{CD} 8^{+} \mathrm{T}$-cell responses as explored in prior work and may also underlie differences in the epitope specificities of the induced antibodies. More study of such platform specific mechanisms will likely be informative.

There are unanswered questions which remain with this approach that should be explored in future work. The glycosylation profiles of in vivo produced trimers, for instance, may be further 
digestion and downstream mass spectrometry analyses of the liberated glycopeptides to establish a correlation between the glycosylation profile and specificities of induced antibodies ${ }^{60}$. Consistency and potency of the nAbs induced by DNA-encoded BG505.MD39 immunizations may be further improved with optimization in the dosing intervals. We observed here that lengthening the third dose from 6 weeks to 16 weeks improved neutralizing responder rate. This observation is consistent with prior work that delayed boost may enhance secondary antibody responses by allowing more time for somatic hypermutations and affinity maturation of germinal center B cells ${ }^{61}$. Clearly, translation of these findings in larger animal species would be of interest for clinical development. This DNA-EP platform has recently shown $>98 \%$ antibody seroconversion rates in a clinical HIV vaccine study ${ }^{62}$ and is being employed to administer a SARS-CoV-2 vaccine ${ }^{23,63,64}$. In addition, the neutralizing immunity directed at C3/V5 elicited by DNA immunization of BG505.MD39 is considerably specific, as we did not observe neutralization breadth across other viruses in the global panel ${ }^{65}$. Therefore, it will be important to consider strategies that may broaden neutralization mediated by the C3/V5 epitope.

Despite these challenges, DNA vaccination still remains as an important strategy for in vivo delivery of NLTs, considering the cost-effectiveness of DNA production, high thermal stability of DNA plasmids ${ }^{66,67}$, the ability of DNA to potentiate in vivo expression of conformationally complex immunogens driven from larger cassettes to induce unique and relevant immune phenotypes ${ }^{31,68}$. The present study opens the door for exciting new studies aiming to deliver complex NLTs, such as cocktails or germlinetargeting immunogens, to rapidly execute pre-clinical NLT studies and to translate NLT immunogens to the clinic.

\section{Methods}

\section{DNA design and plasmid synthesis}

Amino acid sequence for BG505_MD39 based stabilized trimers was obtained from previously published sequence ${ }^{32}$. Control plasmid encoding the gp120_foldon was designed as previously described ${ }^{5}$. The full length unmodified BG505 gp160 sequence was also made using the sequence from GenBank (accession number DQ208458). These sequences were then RNA and codon optimized as well as optimizing for GC content and secondary structure. Additionally, an optimized IgE leader sequence was added to the $\mathrm{C}$ term of the protein to provide efficient processing and secretion. Variable heavy and light chain sequences of isolated murine antibody clones were combined downstream with human IgG1 Fc or Kappa constant light chain. All plasmids were synthesized and cloned (GenScript) into our modified pVAX1 backbone (Inovio Pharmaceuticals). 
The full length BG505 plasmid was used to make point mutations at T332N, T332N S241N, and T332N T456N for pseudotype virus production. Plasmids expressing MG505.H3 Env, HIV backbone $\Delta$ Env (pSG3) and murine leukemia virus control Envelope were obtained from NIH AIDS Reagents Program.

\section{Cell lines, transfection and recombinant antibody purification}

HEK 293 T cells (ATCC) and TZM-bl cells (NIH AIDS Reagents Program) were maintained in DMEM (ThermoFisher) supplemented with $10 \%$ heat inactivated fetal bovine serum (Atlas Biologicals). Expi293F cells (ThermoFisher) were maintained in Expi293 expression medium (ThermoFisher). All cell lines were mycoplasma negative and tested on a regular basis. To produce recombinant HIV monoclonal antibodies for small scale assays, Expi293F cells were transfected following manufacturer's protocol for Expifectamine. Transfection enhancers were added $18 \mathrm{hr}$ after transfection and supernatants were harvested 6 days later. For large scale antibody production, Expi293F cells were transfected using PEI and supernatants were harvested 6 days later. Protein A agarose was used following manufacturer's protocol to purify the IgG. Purity was confirmed with commassie staining of SDS-page gels and concentration was determined using a nanodrop. Supernatant IgG expression was quantified using a standard binding curve from the large-scale purified antibodies in an ELISA.

Pseudotype viruses were produced as previously described ${ }^{69}$. Briefly pseudotyped viruses were produced using HEK 293T cells transfected with 4ug of plasmid expressing the Env of interest and 8ug of plasmid expressing the HIV-1 backbone $\Delta$ Env (pSG3 $\Delta$ Env - NIH AIDS Reagents) using GeneJammer (Aglient). Forty-eight hours after transfection, cell supernatant was harvested, filtered through a $45 \mu \mathrm{m}$ filter, aliquoted and stored at $-80^{\circ} \mathrm{C}$.

\section{Production of trimer}

BG505_MD39-based trimers were expressed in FreeStyle 293F Cells (Invitrogen) and are derived from a low-passage Master Cell Bank and certified mycoplasma free. The trimer-containing supernatants were obtained by centrifuging (4000xg, 25mins) and filtering (0.2um Nalgene Rapid-Flow Filter) the 293F cultures. Trimers were purified from supernatants by lectin purification using lectin beads (Vector Laboratories $7.5 \mathrm{ml}$ beads $/ 1 \mathrm{~L}$ culture) and lectin elution buffer (1M Methyl alpha-D-mannopyranoside). The elution was dialyzed overnight into PBS. The trimers were then purified over a size-exclusion chromatography column (GE S200 Increase) in PBS. The molecular weight and homogeneity of the trimers were confirmed by protein conjugated analysis from ASTRA with data collected from a size-exclusion chromatography-multi-angle light scattering (SEC-MALS) experiment run in PBS using a GE S6 Increase 
column followed by DAWN HELEOS II and Optilab T-rEX detectors. The trimers were aliquoted at $1 \mathrm{mg} / \mathrm{ml}$ and flash frozen in thin-walled PCR tubes prior to use.

\section{Negative Stain EM of Purified Trimers}

SEC purified MD39 trimers were further dialyzed into Tris-buffered saline (TBS). A total of $3 \mu \mathrm{L}$ of purified proteins was adsorbed onto glow discharged carbon-coated Cu400 EM grids. The grids were then stained with $3 \mu \mathrm{L}$ of $2 \%$ uranyl acetate, blotted, and stained again with $3 \mu \mathrm{L}$ of the stain followed by a final blot. Image collection and data processing was performed on a FEI Tecnai T12 microscope equipped with Oneview Gatan camera at $90450 \times$ magnification at the camera and a pixel size of $1.66 \AA$.

\section{Immunization of Mice}

All mice were housed in compliance with the NIH and Wistar's Institutional Animal Care and Use Committee guidelines under IACUC protocol 201214. To test for immunogenicity, 6-8 week old BALB/C mice were immunized with $25 \mathrm{ug}$ of plasmid followed by in vivo electroporation using the CELLECTA ${ }^{\circledR} 3 \mathrm{P}$ adaptive constant current electroporation device (Inovio Pharmaceuticals) or immunized with 25ug of protein co-formulated with Sigma Adjuvant System (RIBI) subcutaneously over the flanks. Mice were immunized at either $0,3,6$ weeks or $0,3,16$ weeks and sacrificed two weeks after final immunization to determine vaccine induced immune responses. Sera were collected at the indicated timepoint through the submandibular vein for assessment of humoral responses. Spleens, iliac, popliteal and inguinal lymph nodes were collected from euthanized animals at specified timepoints for analyses of cellular responses. For NATIVE PAGE and IFA analyses of in vivo produced trimers, BALB/c mice received intramuscular injection of 100ug DNA plasmid encoding either BG505.gp120.Foldon or MD39 co-formulated with 12U hyaluronidase (SigmaAldrich) in the tibialis anterior muscle, followed by IM-EP with the Cellectra 3P device. 4 d.p.i (for NATIVE PAGE analysis) or 7 d.p.i (IFA), mice were euthanized for collection of the injected tibialis anterior muscle.

\section{Mouse muscle staining of in vivo produced antigens}

For muscle staining, 7 days after BALB/c mice were immunized with $100 \mu \mathrm{g}$ DNA plasmid coformulated with $12 \mathrm{U}$ hyaluronidase, the tibialis anterior muscles of the mice were harvested the and preserved in $4 \% \mathrm{PFA} / \mathrm{PBS}$ for $2 \mathrm{~h}$ at room temperature and then stored overnight in $70 \% \mathrm{EtOH} / \mathrm{H}_{2} \mathrm{O}$ at $4{ }^{\circ} \mathrm{C}$. The tissues were then serially dehydrated and blocked in 3\% BSA/PBS for $1 \mathrm{~h}$ at room temperature, followed by overnight staining with $6 \mu \mathrm{g} / \mathrm{mL}$ PGT145 or 3074 (NIH AIDS Reagent). The sections were then 
washed and stained with anti-human Alexa Fluor 488 antibody (Invitrogen), counterstained with 0.5 $\mu \mathrm{g} / \mathrm{mL}$ DAPI (SigmaAldrich) and imaged with a Leica SP5 confocal microscope.

\section{NATIVE PAGE analysis of in vivo produced MD39}

Tibialis anterior muscles of immunized animals were harvested and homogenized in T-PER extraction buffer (Thermo Fisher Scientific) and protease inhibitor (Roche). Muscle homogenates were subsequently concentrated 20x with Amicon Ultra $0.5 \mathrm{~mL}$ centrifugation kits with 3kDA cutoffs (Milipore Sigma) and protein concentrations were quantified with BCA assays (Thermo Fisher Scientific).

For the Coomassie staining, varying amount of MD39 or BG505 gp120 (NIH AIDS Reagent) protein standards were co-incubated with 1x NATIVE sample running buffer and loaded onto 3-12\% Bis-Tris NATIVE PAGE gels (Invitrogen) along with Native Marks. After electrophoresis, the gel was stain with Coomassie Blue R-250 (BioRad) at room temperature for 30 minutes, followed by de-staining with $\mathrm{dH}_{2} \mathrm{O}$.

For the western analysis, $50 \mu \mathrm{g}$ muscle homogenates or protein standards were loaded onto 312\% Bis-Tris NATIVE PAGE gel for electrophoresis. Proteins were subsequently transferred to PVDF membrane from the gels and stained overnight at $4{ }^{\circ} \mathrm{C}$ with $3 \mu \mathrm{g} \mathrm{mL} \mathrm{L}^{-1} 2 \mathrm{G} 12$ antibody (NIH AIDS-Reagent) in Odyssey Blocking Buffer/PBS/0.1\% Tween (LI-COR Biosciences), and 1:10,000 IRDye 800CW goat antihuman IgG (LI-COR Biosciences) in Odyssey Blocking Buffer/0.1\% Tween/0.1\% SDS at room temperature for $1 \mathrm{~h}$, and then scanned with LI-COR Odyssey CLx.

\section{ELISA}

Serological Trimer binding ELISA

Binding titers to trimer were determined by coating plates with $2 \mathrm{ug} / \mathrm{ml}$ of recombinant PGT128 antibody overnight in PBS. After washing, plates were blocked with 5\% skim milk in PBS with $1 \%$ newborn calf serum (NBS) and $0.2 \%$ Tween for $1 \mathrm{~h}$ at RT. Recombinant trimer was added at $4 \mathrm{ug} / \mathrm{ml}$ for $2 \mathrm{hrs}$ at RT. Serum was serially diluted, added to plates and incubated at $37^{\circ} \mathrm{C}$ for $1 \mathrm{hr}$. Antigen and species specific lgG was then detected with across absorbed secondary anti-mouse HRP antibody (Bethyl Laboratories Inc) . Plates were developed for 5 minutes with 1-step ultra TMB (ThermoFisher) and stopped with $2 \mathrm{~N} \mathrm{H}_{2} \mathrm{SO}_{4}$. Absorbance at an optical density (OD) of $450 \mathrm{~nm}$ and $570 \mathrm{~nm}$ was measured on a Synergy2 plate reader (BioTek Instrument). The background 570nm OD was subtracted from the $450 \mathrm{~nm}$ reading.

\section{$\underline{\text { Recombinant Antibody binding ELISA }}$}


Antibody affinity to BG505.MD39 trimer was determined by coating plates with $1 \mathrm{ug} / \mathrm{ml}$ goat antihis6X antibody in 1XPBS for 3hrs at RT. After washing with 1XPBS containing $0.05 \%$ Tween, plates were blocked overnight with 1XPBS containing $0.1 \%$ Tween and 5\% skim milk. Plates were washed and histagged BG505.MD39 was incubated at $1 \mathrm{ug} / \mathrm{ml}$ for $1 \mathrm{~h}$ at RT. Plates were washed and recombinant antibody was serially diluted, added to plates and incubated for $1 \mathrm{~h}$ at RT. After washing the plates, goat anti-human IgG Fc (Bethyl Laboratories Inc) at a dilution of 1:10,000 was incubated for $1 \mathrm{~h}$ at RT. Plates were washed and developed for 5 minutes with 1-step ultra TMB (ThermoFisher) and stopped with $2 \mathrm{~N} \mathrm{H}_{2} \mathrm{SO}_{4}$. Absorbance at an optical density (OD) of $450 \mathrm{~nm}$ and $570 \mathrm{~nm}$ was measured on a Synergy2 plate reader (BioTek Instrument). The background 570nm OD was subtracted from the $450 \mathrm{~nm}$ reading.

\section{Antibody competition ELISA}

96-well half area plates (Corning) were coated at room temperature for 8 hours with $1 \mathrm{ug} / \mathrm{mL}$ PolyRab anti-His antibody (ThermoFisher, PA1-983B), followed by overnight blocking with blocking buffer containing 1x PBS, 5\% skim milk and 0.1\% Tween-20. The plates were then incubated with $1 \mathrm{ug} / \mathrm{mL}$ of histagged BG505.MD39 at room temperature for 1 hour. DLHIV antibodies were serially diluted 3-fold (starting concentration,100ug/mL) with blocking buffer and incubated on the plates for 2 hours. A no competitor control used dilution buffer at this step. C24 and C37 antibodies were biotinylated using Novus Biologicals Lightning-Link rapid type A Biotin antibody labeling kit (NovusBio, 370-0010) according to protocol. The biotinylated antibodies were added to wells at a concentration of $1 \mathrm{ug} / \mathrm{ml}$ and incubated at RT for 1 hour. The plates were further incubated at room temperature for 1 hour with native streptavidinHRP (Abcam, ab7403) at 1:15,000 dilution, followed by addition of TMB substrate (ThermoFisher) and then quenched with $1 \mathrm{M} \mathrm{H} 2 \mathrm{SO}$. Absorbance at $450 \mathrm{~nm}$ and $570 \mathrm{~nm}$ were recorded with a BioTek plate

\section{Neutralization Assay}

Pseudotyped viruses were titered to yield 150, 000 RLU after 48hrs of infection with TZM-BL cells $s^{69}$. Mouse serum was heat inactivated for 15 minutes at $56^{\circ} \mathrm{C}$. Serum or monoclonal antibody controls were serially diluted in 96-well plates and incubated with pseudotyped virus before adding 10,000 TZMBL cells (NIH AIDS Reagent Program) per well with dextran (ThermoFisher). Forty-eight hours after incubation, media was removed and cells were lysed using BriteLite luciferase reagent (Promega). Luminescence was then measured using the Synergy2 plate reader (BioTek Instruments). Serum titer was 


\section{Mouse IFN-gamma Enzyme-linked immunospot assay (ELISpot)}

Ninety-six well filter plates were pre-coated with anti-IFN- $\gamma$ capture antibody (MabTech). Spleens were isolated from mice two week after final immunization. After processing the spleens to obtain a single

514 cell suspension, $2 \times 10^{5}$ cells were added to the blocked plates. Cells were stimulated with overlapping 515 15mer peptide pools for WT BG505 gp160 (5ug/ml per peptide - GenScript). Media alone and concanavalin $516 \mathrm{~A}$ (Invitrogen) were used as negative and positive controls respectively. After $18 \mathrm{hrs}$ of stimulation at $37^{\circ} \mathrm{C}$, 517 the plates were washed, and detection antibody (R4-6A2-biotin) was added for $2 \mathrm{hrs}$ at RT. Plates were 518 then washed and the Streptavidin-ALP antibody was added for $1 \mathrm{hr}$ at RT. Plates were then developed 519 using the BCIP/NBT-plus for 10 minutes. Plates were then scanned and counted using CTL-ImmunoSpot ${ }^{\circ}$ 520 S6 FluoroSpot plate reader (Cellular Technology Limited CTL).

Flow cytometry-based assays Intracellular cytokine staining

For intracellular cytokine staining, $2 \times 10^{6}$ splenocytes were stimulated in the presence of protein 525 transport inhibitor, GolgiStop ${ }^{\mathrm{TM}}$ GolgiPlug $^{\mathrm{TM}}$ (BD Bioscience) with the same peptide pools as the ELISpots. 526 Media alone and phorbol 12-myristate 13-acetate (PMA) and ionomycin stimulations (BD Bioscience) 527 were used as negative and positive controls respectively. To test for degranulation of cells, FITC anti528 CD107a (Biolegend) antibody was also added during stimulation. After 6hrs, cells were washed and 529 stained with LIVE/DEAD violet. Surface staining was then added containing BV510 anti-CD4, APC-Cy7 anti530 CD8, BV711 anti-CD62L and AF700 anti-CD44 (Biolegend). After 30 minutes incubation, cells were spun, 531 washed, and fixed using the CytoPerm CytoWash kit following manufacturer's protocol (BD Bioscience). 532 Intracellular staining was then prepared using APC anti-IFN $\gamma$, BV650 anti-TNF $\alpha$, PE-Cy7 anti-IL2, and PE533 Cy5 anti-CD3 (Biolegend). All data was collected on a modified LSRIl flow cytometer (BD Bioscience) 534 followed by analysis with FlowJo software (BD Bioscience). Tfh and GCB-cell staining

Single cell suspension was generated from the spleen as described in the prior section, and from 537 the lymph nodes by applying pressure on the tissues to pass through 40 um strainers. Single cell 538 suspensions were washed once with PBS, and then stained with live dead dye (fluorescent violet reactive, 539 Thermo Fisher) diluted 333-fold in PBS at room temperature for 10 minutes, followed by another wash in 540 PBS. The cells were then incubated with mouse Fc-block (anti-mouse CD16/32, BioLegend) in 1\% FBS/PBS 541 at room temperature for additional 5 minutes, followed by incubation with antibody mixtures (anti-mouse 542 GL7-FITC, anti-mouse CD4 BV510, anti-mouse CD44 AF700, anti-mouse PD1 PE-Cy7 and anti-mouse CXCR5 
biotin, BioLegend, each diluted 1:200 in 1\% FBS/PBS) without washing for an additional 40 minutes at room temperature. The cells were washed and then incubated with Streptavidin-APC at $0.5 \mathrm{ug} / \mathrm{mL}$ in $1 \%$ FBS/PBS for 20 minutes at room temperature. The cells were then washed again and resuspended in $1 \%$ FBS/PBS for flow analysis with an LSRII 18-color instrument.

\section{Antigen-specific B-cell sorting}

To generate FITC and PE labelled MD39 tetramers, biotinylated avi-tagged MD39 produced as previously mentioned were incubated with molar ratios of Streptavidin-FITC or Streptavidin-PE (BioLegend) for 30 minutes at room temperature ${ }^{8,51}$. Single cell suspensions from spleen or lymph nodes of the animals were first washed with PBS, then stained with live dead dye (fluorescent violet reactive, Thermo Fisher) diluted in PBS at room temperature for 10 minutes, followed by addition of mouse Fcblock (anti-mouse CD16/32, BioLegend) diluted 1:100 in 1\% FBS/PBS and incubation at room temperature for additional 30 minutes. The cells were washed, and then incubated with 5ug/mL Streptavidin-MD39FITC and Streptavidin-MD39-PE at room temperature for 45 minutes. Antibody mixtures (anti-mouse CD19-PE-Cy7, Anti-mouse IgD APC-Cy7 and Anti-mouse IgM BV711, BioLegend, each diluted 1:200 in 1\% FBS/PBS) were then added to the cells without any intermediate washing steps, followed by incubation for another 45 minutes at room temperature. The cells were washed once with $1 \%$ FBS/PBS, and then resuspended at 10 million/mL concentration in 1\% FBS/PBS for sorting with the FACS ARIA II instrument. CD19+ IgM- IgD- MD39-Tetramer-FITC+ MD39-Tetramer-PE+ cells were then sorted in bulk into $1.5 \mathrm{~mL}$ Eppendorf tube containing 1\% FBS/PBS for down-stream cDNA prep with 10x genomics.

cDNA library prep of sorted B-cells with 10x genomics, enrichment of heavy and light chain sequences and next-generation sequencing

Sorted and viable single B cells from each mouse sample were uniquely barcoded using the $10 x$ chromium single-cell platform, and complementary DNA (cDNA) libraries were prepared from enriched mouse VDJ regions for Next Generation Sequencing according to the manufacturer's protocol (Chromium Next GEM Single Cell V(D)J Reagent Kits v1.1, 10x Genomics, USA). Cell suspensions of each sample, reverse transcription master mix, and partitioning oil were loaded on a lane of a single-cell " $G$ " chip with a targeted cell output of 2,000 cells per library and then run on the Chromium Controller. Reverse transcription was performed within the droplets at $53^{\circ} \mathrm{C}$ for $45 \mathrm{~min}$ and newly synthesized cDNA was amplified for 16 cycles on a Veriti Thermal Cycler (Thermofisher, USA). cDNA size selection was performed using SPRIselect beads (Beckman Coulter, USA) at a ratio of SPRIselect reagent volume to sample volume of 0.6. cDNA was analyzed on an Agilent Bioanalyzer High Sensitivity DNA chip (Agilent, USA) for 
575 qualitative and quantitative control purposes. Enrichment of the mouse V(D)J region was done using 576 primers targeting the Illumina P5 oligo sequence and the mouse constant region. Two rounds of targeted 577 PCRs were done at $67^{\circ} \mathrm{C}$ for $30 \mathrm{~min}$. for 6 and $8 \mathrm{cycles}$, respectively. SPRIselect bead clean-up was done in 578 between and after the $V(D) J$ enrichment PCRs. $V(D) J$ enriched cDNA was analyzed on an Agilent 579 Bioanalyzer High Sensitivity DNA chip (Agilent, USA) for qualitative and quantitative control purposes. $580 \mathrm{~V}(\mathrm{D}) \mathrm{J}$ enriched cDNA was fragmented using the proprietary fragmentation enzyme blend for $2 \mathrm{~min}$ at $32^{\circ} \mathrm{C}$, 581 followed by end-repair and A-tailing at $65^{\circ} \mathrm{C}$ for $30 \mathrm{~min}$. Sequencing adaptors were ligated to the cDNA at $58220^{\circ} \mathrm{C}$ for $15 \mathrm{~min}$. and after a round of post-ligation SPRIselect bead clean-up, V(D)J enriched cDNA was 583 amplified for 9 cycles using a sample-specific index oligo as a primer. A final round of size selection using 584 SPRIselect beads followed. Final library size and quantity was determined using an Agilent Bioanalyzer 585 High Sensitivity DNA chip and a Qubit dsDNA High Sensitivity Assay kit (Thermofisher, USA), respectively. Additional library quantification was done using the Kapa Library Quantification kit for Illumina Libraries 587 (Roche, USA). cDNA libraries were sequenced on a NextSeq 500 Illumina platform using the 300bp Mid 588 Output sequencing kit (Illumina, USA), at a sequencing configuration of 150 base pair (bp) on read1 and $589150 \mathrm{bp}$ on read2.

\section{Modeling Murine Fabs}

Structural models of C05, C24 and C33 were generated using an anti-citrullinated protein antibody 592 (PDB-ID: 5mu0) that uses the same germline pair (IGHV6-6/IGKV3-2) as a template. Amino acids sequences of C05, C24 and C33 were each aligned to the 5mu0 Fab using default alignment algorithm in Geneious software. A Rosetta fixed backbone simulations employing the beta score function was performed to mutate positions with different SHM changes in C05, C24 and C33. The variable loops were modeled using Rosetta Remodel to the appropriate sequences for C05, C24 and C33. Finally, Rosetta Relax protocol was performed using backbone constraints to minimize and polish the models.

\section{Complexing of Trimer with Murine Fab and CryoEM of MD39-Fab complex}

600 Murine variable chains were cloned onto human constant IgG domains and expressed in Expi293F cells 601 following the recombinant antibody purification protocol described above. The purified antibodies were digested into Fabs using a Thermo Scientific Pierce Fab preparation kit. Fab purity was determined by a non-reducing SDS-PAGE gel and concentrations were taken by a nanodrop. Cryo-EM specimens were prepared similarly for complexes between C05, C24 and C33 Fab and BG505.MD39. Complexes were

605 formed at a 2:1 molar ratio of Fab to antigen binding site overnight at 4C. Complex was purified by size606 exclusion chromatography employing an S6i column (GE Healthcare). Sample aliquot was deposited on a 
holey carbon grid (1.2/1.3um configuration), blotted and plunge frozen in a Vitrobot Mark IV (Thermo Fisher Scientific). Vitrified cryo-EM specimens were introduced into a TFS Talos Arctica (Thermo Fisher Scientific) and data was recorded using a TFS Falcon 3 camera (Thermo Fisher Scientific). The resulting data were processed using standard workflows in Relion $3.0^{70}$.

\section{Statistical analysis}

All statistics and calculations were performed using GraphPad Prism 8.0. EC 50 and $E C_{70}$ concentrations were calculated using a non-linear regression model. I $\mathrm{C}_{50}$ values were computed with a non-linear regression model of percentage neutralization vs log reciprocal serum dilution. Power analysis was performed with $\mathrm{R}$ based on our preliminary data to determine the smallest sample size that would allow us to achieve a power of 0.9 with a pre-set $\alpha$-value of 0.05 . Each individual data point was sampled independently. Two-tailed Mann Whitney Rank tests were used to compare differences between groups with correction for multiple-comparisons. P-values were all calculated in GraphPad PRISM, and p-values less than 0.05 are considered as significant.

\section{Figure Legends}

Figure 1. DNA/EP delivery of BG505.MD39 can enable in vivo expression of conformationally complex NLT. (A) SEC trace of lectin-column purified BG505.MD39 that has been sequence optimized for in vivo expression. (B) Antigenic profile of sequence optimized BG505.MD39 in terms of ELISA binding to V2-apex directed bNAb PGT145 and v3-directed non-nAb 3074. (C) nsEM image of SEC purified sequence optimized BG505.MD39. (D) Coomassie staining for the migrations of the purified in vitro expressed BG505.MD39 and BG505 gp120 protein standards on 3-12\% Bis-Tris NATIVE PAGE gels. (E) 2G12-based western analysis to compare the migrations of the in vivo produced BG505.MD39 in muscle homogenates from mice treated with DNA-encoded BG505.MD39 (harvested 4 d.p.i) to those of in vitro produced BG505.MD39 and BG505 gp120 on a 3-12\% Bis-Tris NATIVE PAGE gel. (F) Immuno-fluorescence analyses of muscle sections from mice treated intramuscularly with DNA-encoded BG505.MD39 or BG505.gp120.foldon (harvested 7 d.p.i) in terms of binding to bNAb PGT145 or non-nAb 3074.

Figure 2. DNA immunization of BG505.MD39 induced stronger T-cell responses and NAb responses than protein immunization in BALB/c mice. All mice in this panel were immunized at Wks 0, 3, 6 with 25ug DNA or $25 \mathrm{ug}$ RIBI-co-formulated protein and euthanized 2 weeks post the final vaccination for cellular analyses. (A and B) Comparison of BG505 Env specific cellular responses in naïve mice, or mice immunized 
with pVAX plasmid backbone, RIBI-co-formulated protein BG505.MD39, or DNA-encoded BG505.MD39 by IFNY ELIspot assay (A) or intracellular cytokine staining (B). (C) Frequencies of CXCR5+PD1+ Tfh cells amongst CD4+CD44+ cells in the draining lymph nodes in naïve mice or 10 days post pVAX1, protein MD39 or DNA BG505.MD39 immunization. (D) Frequencies of GL7+ GC B cells amongst CD19+ B cells in the draining lymph nodes in naïve mice or 10 days post pVAX1, protein BG505.MD39 or DNA BG505.MD39 immunization. (E) Trimer-specific binding antibody responses induced in mice vaccinated with protein or DNA-encoded BG505.MD39 post the first, second and third immunization as determined by ELISA. (F) Frequencies of mice that developed autologous BG505.T332N neutralizing antibodies $\left(\right.$ ID $_{50}$ titers greater than 1:45) in naïve mice or mice immunized with pVAX1, protein BG505.MD39 or DNA-encoded BG505.MD39 two weeks post the final vaccination. Two independent experiments were performed for each panel in the figure. $\mathrm{N}=10$ mice/group for protein or DNA encoded BG505.MD39, N=5 mice/group for naïve mice or pVAX1 treated mice (A, B, E, F); N=5 mice/group (C-D); each dot or line represents an animal. Error bar represents standard deviation. Two-tailed Mann-Whitney Rank test used to compare groups; $\mathrm{p}$ values were adjusted for multiple comparison for panels (A-D); ns: not significant.

Figure 3. Prolonging the duration between the second and third immunizations increased frequencies of responders who developed autologous Tier 2 NAbs. Mice in this panel were immunized either with a short scheme (Wks 0,3,6) or a long scheme (Wks 0, 3, and 16) with 25ug DNA used at each vaccination. The mice were euthanized 2 weeks post the final vaccination for cellular analyses. (A) Vaccination scheme used in this study. (B and C) Comparison of BG505 Env specific cellular responses in naïve mice, mice immunized with pVAX1 plasmid backbone, or those immunized with DNA-encoded BG505.MD39 using either the short or the long scheme by IFNY ELISpot assay (B) or intracellular cytokine staining (C). (D and E) Time course of trimer-specific binding antibodies in naïve mice or mice vaccinated with DNA-encoded BG505.MD39 using either the short scheme (D) or the long scheme (E) as determined by ELISA. (F) Comparison of trimer specific binding antibodies at the final timepoint (two weeks post the third vaccination) in mice immunized with DNA-encoded BG505.MD39 using either the short or the long scheme. (G) Frequencies of mice that developed autologous BG505.T332N neutralizing antibodies (ID ${ }_{50}$ titers greater than 1:45) in naïve mice, mice immunized with pVAX1, or mice immunized with DNAencoded BG505.MD39 using either the short or the long scheme two weeks post the final vaccination. Two independent experiments were performed for each panel in the figure. $\mathrm{N}=5$ mice/group for ( $\mathbf{A}$ - F); $\mathrm{N}=10$ mice/group (G); each dot or line represents an animal. Error bar represents standard deviation. Twotailed Mann-Whitney Rank test used to compare groups; $p$-values were adjusted for multiple comparison for panels (B-C); ns: not significant. 
Figure 4. Screening with engineered BG505.T332N pseudo-viruses with modified glycosylation sites mapped induced murine NAb to the V5-loop of Envelope. Mice in this panel were immunized with variations of DNA-encoded BG505.MD39 at Wks 0, 3 and 16 with 25ug DNA used at each vaccination and identified to possess potent BG505.T332N neutralizing activities 2 weeks post the final vaccination. (A and B) Rosetta modeling to demonstrate the locations of the engineered glycosylation sites (A) and the V5 loop (B) on the NLT. (C) Neutralization ID50 titers of heat-inactivated mice sera against listed pseudovirus isolates. (D) Sequence alignment of the V5 loops for BG505.W6M.ENV.C2 and MG505.WOM.ENV.H3.

Figure 5. Isolation of trimer specific monoclonal antibodies from an animal (272.8) with potent neutralization activity against BG505.T332N post vaccinations with DNA-encoded BG505.MD39. (A) Vaccination and tissue collection scheme for 272.8. (B) Trimer-specific binding antibody responses in 272.8 two weeks post the final vaccination. (C) Autologous BG505.T332N neutralizing activity induced in 272.8 two weeks post the final vaccination. (D) Gating strategy used to isolate antigen-specific CD19+ IgDIgM- BG505.MD39-Tetramer-FITC+ BG505.MD39-Tetramer-PE+ B-cells from spleen and lymph nodes of 272.8 two weeks post the final vaccination. (E) Characteristics of isolated murine monoclonal antibodies in terms of germline VH and VL gene usage.

Figure 6. Isolated trimer-specific murine MAbs neutralized BG505.T332N pseudovirus in a V5dependent fashion. (A) Trimer specific binding of 5 isolated murine MAbs and PGT128 in Expi293F transfection supernatant. (B - D) Neutralization of BG505.T332N (B), BG505.T332N.T465N (C) and MLV (D) pseudo-viruses with varying concentrations of murine trimer-specific MAbs or PGT128. (E) Determined IC50 values in ug/mL of trimer-specific murine MAbs for BG505.T332N, BG505.T332N.T465N or MLV. F. Amino acid alignments of the HCDR3 and LCDR3 regions of the identified BG505.MD39-specific murine antibody clones as compared to the corresponding germline sequences. G. Predicted Rosetta structures of the Fabs of clones C05, C24 and C33, positions mutated from the germline sequences are displayed in red.

Figure 7. Cryo-EM based structural characterization of Fabs of murine nAbs bound to BG505.MD39. (A) Cryo-EM structure of BG505.MD39 in complex with murine neutralizing Fab C24 (BG505.MD39 in grey surface and C24 Fab in orange surface). (B) Interface of structure in (A) with Env gp120 epitope shown as cartoons and lines. (C) Top view of three cryo-EM structures: C24 (orange), C05 (red) and C33 (magenta) (D) Structural alignment using density of complexed Env for four C3/V5 targeting abs from a SHIVinfected NHP (blue), a NLT-immunized rabbit (white), a NLT-vaccinated and challenged NHP (grey) and murine nAbs C24 (orange). Both side and top view are shown and Env is shown in green. 
701

702

703

704

705

706

707

708

709

710

711

712

713

714

715

716

717

718

719

720

721

722

723

724

725

726

727

728

729

730

Table 1. Detailed characterization of isolated murine BG505.MD39-specific clones in terms of VH, VJ, VD, VK, VK, VJ gene usage, HCDR3 and LCDR3 lengths.

\section{Supplemental Figure Legends}

Supplemental Figure S1. Full antigenic profile of in vitro expressed MD39 vs BG505.gp120_foldon (related to Figure 1). (A) Binding of MD39 to HIV bNAbs PGT145, PGT151, 35022, VRC01, N6, PGT121 or non-NAbs B6, 3074, 4025, 17B. (B) Binding of BG505.gp120_foldon to HIV bNAbs and non-NAbs as in (A). (C) Quantification of binding of MD39 or BG505.gp120_foldon to HIV bNAbs or non-NAbs in terms of Area Under Curve (AUC).

Supplemental Figure S2. Gating scheme used to determine cytokine positive $\mathrm{CD4}^{+}$or $\mathrm{CD}^{+} \mathrm{T}$ cells following peptide stimulation (related to Figure 2 and 3 ).

Supplemental Figure S3. DNA-encoded BG505.MD39 but not protein BG505.MD39 induced polyfunctional T-cell responses in BALB/c mice (related to Figure 2). Mice were immunized with 25ug of either DNA-encoded BG505.MD39 or RIBI-co-formulated protein BG505.MD39 at weeks 0, 3, 6 and euthanized at week 8 for cellular analysis. (A - B) ICS analyses of co-expression of IFNY, TNF $\alpha$ and IL-2 cytokines in $\mathrm{CD}^{+}(\mathrm{A})$ or $\mathrm{CD}^{+}$(B) T cells following stimulation with BG505 Envelope specific peptide pools. Two independent experiments were performed for each panel in the figure with similar findings. $\mathrm{N}=10$ mice/group. Error bar represents standard deviation.

Supplemental Figure S4. DNA-encoded BG505.MD39 vaccination induced both Tfh and GC B cell responses in BALB/c mice (related to Figure 2). Mice were immunized with 25ug of DNA-encoded BG505.MD39 on Day 0 and euthanized sequentially on Days 4, 7 and 10 for analyses of Tfh and GC B cells. (A) Comparison of flow plots of CXCR5+PD1+ cells amongst CD4+CD44+ cells in the iliac, inguinal, popliteal and spleen of naïve mice versus mice immunized with DNA-encoded BG505.MD39 10 d.p.i. (B) Kinetics of Tfh and GC B cell responses in the draining lymph nodes or spleens of mice vaccinated with DNA-encoded BG505.MD39. One independent experiment was performed for each panel in the figure. Each line represents an animal. $\mathrm{N}=3$ mice/group.

Supplemental Figure S5. Long immunization scheme with DNA-encoded BG505.MD39 improved polyfunctional $\mathrm{CD}^{+}$T-cell responses, while short immunization scheme improved polyfunctional CD8 ${ }^{+}$ T-cell responses in mice (related to Figure 3). Mice in this panel were immunized either with a short scheme (Wks 0, 3, 6) or a long scheme (Wks 0, 3, and 16) with 25ug DNA used at each vaccination. The 
mice were euthanized 2 weeks post the final vaccination for cellular analyses. (A - B) ICS analyses of coexpression of IFN $\gamma$, TNF $\alpha$ and IL-2 cytokines in $\mathrm{CD}^{+}(\mathbf{A})$ or $\mathrm{CD}^{+}$(B) T cells following stimulation with BG505 Envelope specific peptide pools in mice immunized with DNA-encoded BG505.MD39 using either the short or the long scheme. Two independent experiments were performed for each panel in the figure with similar findings. $\mathrm{N}=5$ mice/group. Error bar represents standard deviation.

Supplemental Figure S6. Sequence features of recovered murine antibody clones (related to Figure 5). (A) Demonstration of the barcode frequencies by clonotypes (Left) or IGH HCDR3 identities (Right). (B) HCDR3 features of the 25 recovered MAb clones (left) or the 6 clones with IGHV6-6 germline VH gene usage (Right).

Supplemental Figure S7. Further characterizations of BG505.MD39-specific murine antibody clones (related to Figure 6). (A-C) Binding of all isolated murine mAb clones to BG505.MD39 (A) or gp120_foldon (B) as determined by ELISA, and respective measured $\mathrm{EC}_{50}$ values (C). (D) Reducing SDS PAGE analysis of BG505.MD39-specific MAb clones C05, C22, C24, C33, and C37 in comparison with PGT128 or backbone pVAX vector transfection supernatants. (E) Determination of the antibody concentrations from the transfection supernatants based on BG505.MD39 binding in comparison with purified protein standards. (F) Comparison of neutralization potential of PGT128 with BG505.T332N pseudo-virus versus BG505.T332N.T465N pseudo-virus.

Supplemental Figure S8. Additional sequence features of recovered murine antibody clones (related to Figure 6). Comparisons of the heavy and light chain sequences of the 5 BG505.MD39-specific MAb clones by number of mutations (A) and positions for the mutations (B).

Supplemental Figure S9. Competition ELISA of biotinylated C24 with non-biotinylated C05, C22, C33, C37 and PGT128 in terms of binding to MD39 (related to Figure 6). (A) Degree of binding of biotinylated C24 to MD39 in the presence of varying concentrations of non-biotinylated C05, C22, C24, C33, C37 and control buffer. (B) Degree of blocking of biotinylated C24 binding to MD39 by each of the aforementioned antibodies, as calculated from (A). Two technical replicates were determined for each experimental condition; error bar represents standard deviation.

\section{Supplemental Table Legends}


Supplemental Table S1. BG505.T332N or MLV ID50 neutralization titers in the sera of animals immunized with 25ug RIBI co-formulated protein BG505.MD39 or DNA-encoded BG505.MD39 at Weeks $0,3,6$ two weeks post the final vaccination.

Supplemental Table S2. BG505.T332N or MLV ID50 neutralization titers in the sera of naïve mice or animals immunized with 25ug DNA-encoded BG505.MD39 at Weeks 0, 3, 6 (short scheme) or Weeks 0, 3, 16 (long scheme) two weeks post the final vaccination.

\section{Funding sources}

This research is supported by NIH IPCAVD Grant U19 Al109646-04 and "Study and Development of Consensus Immunogens in the area of Viral Vaccines" grant from Inovio Pharmaceuticals awarded to D.B.W, W. W. Smith Charitable Trust awarded to D.W.K, and by Wistar Monica H.M. Shander Memorial Fellowship awarded to Z.X.

\section{Conflicts of interests}

M.C.W., D.B.W and D.W.K. have a pending patent with regard to in vivo assembly of trimers using DNA delivery. M.C.W, K.E.B., and L.M.H are employees of Inovio Pharmaceuticals and as such receive salary and benefits including ownership of stock and stock options from the company. D.B.W. has received grant funding, participates in industry collaborations, has received speaking honoraria, and has received fees for consulting, including serving on scientific review committees and board series. Remuneration received by D.B.W. includes direct payments, stock or stock options, and in the interest of disclosure he notes potential conflicts associated with his work with Inovio and possible others.

\section{$\underline{\text { Data and Reagents availability }}$}

No accession codes and identifiers are generated for this manuscript. Raw data is included in the separate excel document where appropriate. All raw data and reagents generated in this paper are available upon request to the corresponding author Dr. Daniel W. Kulp (dwkulp@wistar.org).

\section{$\underline{\text { Acknowledgements }}$}


786 We would like to thank Animal Facility staff at Wistar Institute for providing care to the animals. We thank

787 Imaging Facility Core at Wistar Institute for assistance with in vivo imaging experiment. We thank 788 Histotechnology Core at Wistar Institute for assistance with sectioning/ preparation of sample specimens. 789 We thank the Flow Core at the Wistar Institute for assistance on the flow experiments. The following 790 reagent was obtained through the NIH AIDS Reagent Program, Division of AIDS, NIAID, NIH: Anti-HIV-1 791 gp120 Monoclonal (PGT145) from IAVI (cat\# 12703), Anti-HIV-1 gp120 Monoclonal (3074) from Dr. Susan 792 Zolla-Pazne, Anti-HIV-1 gp120 Monoclonal (2G12) from Polymun Scientific and Anti-HIV-1 gp120 793 Monoclonal (PGT128) from IAVI.

\section{$794 \quad$ Author contributions}

795 Z.X., M.C.W., D.B.W and D.W.K. conceptualized the project. Z.X., M.C.W., S.W., D.B.W., and D.W.K. 796 planned the experiments. Z.X., M.C.W., M.P., S.W., N.C., E.T.R., Y.W., I.P., A.O. conducted the experiments. 797 F.Z., E.L.R., K.S., T.S., L.G., T.S., J.M., K.E.B. and L. H. contributed crucial reagents or equipment. Z.X., 798 M.C.W., M.P., S.W., N.C., D.B.W, and D.W.K analyzed the data. Z.X., M.C.W., M.P., S.W., N.C., D.B.W. and 799 D.W.K. wrote the paper. 
801

8021 Barouch, D. H. Challenges in the development of an HIV-1 vaccine. Nature 455, 613-619,

803 doi:10.1038/nature07352 (2008).

8042 Andrabi, R., Bhiman, J. N. \& Burton, D. R. Strategies for a multi-stage neutralizing antibody-

805 based HIV vaccine. Curr Opin Immunol 53, 143-151, doi:10.1016/j.coi.2018.04.025 (2018).

8063 Nabel, G. J., Kwong, P. D. \& Mascola, J. R. Progress in the rational design of an AIDS vaccine.

$807 \quad$ Philos Trans R Soc Lond B Biol Sci 366, 2759-2765, doi:10.1098/rstb.2011.0096 (2011).

8084 Pejchal, R. \& Wilson, I. A. Structure-based vaccine design in HIV: blind men and the elephant?

809

810 Curr Pharm Des 16, 3744-3753, doi:10.2174/138161210794079173 (2010).

5 AlSalmi, W. et al. A New Approach to Produce HIV-1 Envelope Trimers: BOTH CLEAVAGE AND PROPER GLYCOSYLATION ARE ESSENTIAL TO GENERATE AUTHENTIC TRIMERS. J Biol Chem 290, 19780-19795, doi:10.1074/jbc.M115.656611 (2015).

812

813

Sliepen, K., van Montfort, T., Melchers, M., Isik, G. \& Sanders, R. W. Immunosilencing a highly

814

815 immunogenic protein trimerization domain. J Biol Chem 290, 7436-7442, doi:10.1074/jbc.M114.620534 (2015).

816

Pugach, P. et al. A native-like SOSIP.664 trimer based on an HIV-1 subtype B env gene. J Virol 89,

817

818

819

820

821

822

823

824

825

826

827

828

829 3380-3395, doi:10.1128/JVI.03473-14 (2015).

8 Kulp, D. W. et al. Structure-based design of native-like HIV-1 envelope trimers to silence nonneutralizing epitopes and eliminate CD4 binding. Nat Commun 8, 1655, doi:10.1038/s41467017-01549-6 (2017).

9 Wang, Q. et al. Stabilized diverse HIV-1 envelope trimers for vaccine design. Emerg Microbes Infect 9, 775-786, doi:10.1080/22221751.2020.1745093 (2020).

10 Rawi, R. et al. Automated Design by Structure-Based Stabilization and Consensus Repair to Achieve Prefusion-Closed Envelope Trimers in a Wide Variety of HIV Strains. Cell Rep 33, 108432, doi:10.1016/j.celrep.2020.108432 (2020).

$11 \mathrm{Xu}, \mathrm{Z}$. \& Kulp, D. W. Protein engineering and particulate display of B-cell epitopes to facilitate development of novel vaccines. Curr Opin Immunol 59, 49-56, doi:10.1016/j.coi.2019.03.003 (2019).

830

831

832

833

834

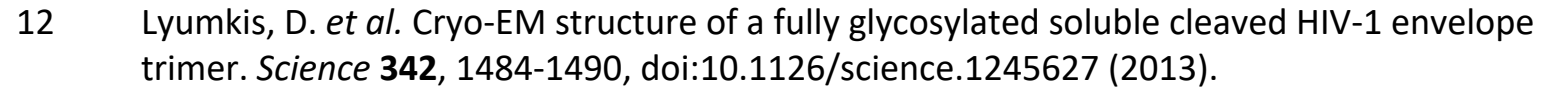

13 Pauthner, M. G. et al. Vaccine-Induced Protection from Homologous Tier 2 SHIV Challenge in Nonhuman Primates Depends on Serum-Neutralizing Antibody Titers. Immunity 50, 241-252 e246, doi:10.1016/j.immuni.2018.11.011 (2019).

14 Klasse, P. J. et al. Sequential and Simultaneous Immunization of Rabbits with HIV-1 Envelope Glycoprotein SOSIP.664 Trimers from Clades A, B and C. PLoS Pathog 12, e1005864, doi:10.1371/journal.ppat.1005864 (2016).

15 Bianchi, M. et al. Electron-Microscopy-Based Epitope Mapping Defines Specificities of Polyclonal Antibodies Elicited during HIV-1 BG505 Envelope Trimer Immunization. Immunity 49, 288-300 e288, doi:10.1016/j.immuni.2018.07.009 (2018).

841 Sarkar, S. \& Heise, M. T. Mouse Models as Resources for Studying Infectious Diseases. Clin Ther 41, 1912-1922, doi:10.1016/j.clinthera.2019.08.010 (2019).

843 $\mathrm{Hu}$, J. K. et al. Murine Antibody Responses to Cleaved Soluble HIV-1 Envelope Trimers Are Highly

17 Restricted in Specificity. J Virol 89, 10383-10398, doi:10.1128/JVI.01653-15 (2015).

845 Escolano, A. et al. Immunization expands B cells specific to HIV-1 V3 glycan in mice and macaques. Nature 570, 468-473, doi:10.1038/s41586-019-1250-z (2019). 


\begin{tabular}{|c|c|c|}
\hline $\begin{array}{l}846 \\
847\end{array}$ & 19 & $\begin{array}{l}\text { Nogal, B. et al. HIV envelope trimer-elicited autologous neutralizing antibodies bind a region } \\
\text { overlapping the N332 glycan supersite. Sci Adv 6, eaba0512, doi:10.1126/sciadv.aba0512 (2020). }\end{array}$ \\
\hline 848 & 20 & Klasse, P. J. et al. Epitopes for neutralizing antibodies induced by HIV-1 envelope glycoprotein \\
\hline 849 & & BG505 SOSIP trimers in rabbits and macaques. PLoS Pathog 14, e1006913, \\
\hline 850 & & doi:10.1371/journal.ppat.1006913 (2018). \\
\hline 851 & 21 & Jones, A. T. et al. A Trimeric HIV-1 Envelope gp120 Immunogen Induces Potent and Broad Anti- \\
\hline 852 & & V1V2 Loop Antibodies against HIV-1 in Rabbits and Rhesus Macaques. J Viro/ 92, \\
\hline 853 & & doi:10.1128/JVI.01796-17 (2018). \\
\hline $\begin{array}{l}854 \\
855\end{array}$ & 22 & $\begin{array}{l}\text { He, L. et al. HIV-1 vaccine design through minimizing envelope metastability. Sci Adv 4, } \\
\text { eaau6769, doi:10.1126/sciadv.aau6769 (2018). }\end{array}$ \\
\hline $\begin{array}{l}856 \\
857\end{array}$ & 23 & $\begin{array}{l}\text { Kutzler, M. A. \& Weiner, D. B. DNA vaccines: ready for prime time? Nat Rev Genet 9, 776-788, } \\
\text { doi:10.1038/nrg2432 (2008). }\end{array}$ \\
\hline 858 & 24 & Tebas, P. et al. Intradermal SynCon(R) Ebola GP DNA Vaccine is Temperature Stable and Safely \\
\hline 859 & & Demonstrates Cellular and Humoral Immunogenicity Advantages in Healthy Volunteers. J Infect \\
\hline 860 & & Dis, doi:10.1093/infdis/jiz132 (2019). \\
\hline 861 & 25 & Tebas, P. et al. Safety and Immunogenicity of an Anti-Zika Virus DNA Vaccine - Preliminary \\
\hline 862 & & Report. N Engl J Med, doi:10.1056/NEJMoa1708120 (2017). \\
\hline 863 & 26 & Modjarrad, K. et al. Safety and immunogenicity of an anti-Middle East respiratory syndrome \\
\hline 864 & & coronavirus DNA vaccine: a phase 1 , open-label, single-arm, dose-escalation trial. Lancet Infect \\
\hline 865 & & Dis, doi:10.1016/S1473-3099(19)30266-X (2019). \\
\hline 866 & 27 & DeRosa, S. et al. Robust antibody and cellular responses induced by DNA-only vaccination for \\
\hline 867 & & HIV. JCI Insight, doi:10.1172/jci.insight.137079 (2020). \\
\hline 868 & 28 & Xu, Z. et al. Synthetic DNA delivery by electroporation promotes robust in vivo sulfation of \\
\hline 869 & & broadly neutralizing anti-HIV immunoadhesin eCD4-Ig. EBioMedicine 35, 97-105, \\
\hline 870 & & doi:10.1016/j.ebiom.2018.08.027 (2018). \\
\hline 871 & 29 & Wise, M. C. et al. In vivo delivery of synthetic DNA-encoded \\
\hline 872 & & neutralizing activity. J Clin Invest, doi:10.1172/JCI132779 (2020). \\
\hline 873 & 30 & Patel, A. et al. In Vivo Delivery of Synthetic Human DNA-Encoded Monoclonal Antibodies Protect \\
\hline 874 & & against Ebolavirus Infection in a Mouse Model. Cell Rep 25, 1982-1993 e1984, \\
\hline 875 & & doi:10.1016/j.celrep.2018.10.062 (2018). \\
\hline 876 & 31 & Xu, Z. et al. In Vivo Assembly of Nanoparticles Achieved through Synergy of Structure-Based \\
\hline 877 & & Protein Engineering and Synthetic DNA Generates Enhanced Adaptive Immunity. Adv Sci \\
\hline 878 & & (Weinh) 7, 1902802, doi:10.1002/advs.201902802 (2020). \\
\hline 879 & 32 & Steichen, J. M. et al. HIV Vaccine Design to Target Germline Precursors of Glycan-Dependent \\
\hline 880 & & Broadly Neutralizing Antibodies. Immunity 45, 483-496, doi:10.1016/j.immuni.2016.08.016 \\
\hline 881 & & (2016). \\
\hline 882 & 33 & Kim, H. et al. Gene therapy using plasmid DNA-encoded anti-HER2 antibody for cancers that \\
\hline 883 & & overexpress HER2. Cancer Gene Ther 23, 341-347, doi:10.1038/cgt.2016.37 (2016). \\
\hline 884 & 34 & Andrabi, R. et al. Identification of Common Features in Prototype Broadly Neutralizing \\
\hline 885 & & Antibodies to HIV Envelope V2 Apex to Facilitate Vaccine Design. Immunity 43, 959-973, \\
\hline 886 & & doi:10.1016/j.immuni.2015.10.014 (2015). \\
\hline 887 & 35 & Hioe, C. E. et al. Anti-V3 monoclonal antibodies display broad neutralizing activities against \\
\hline 888 & & multiple HIV-1 subtypes. PLoS One 5, e10254, doi:10.1371/journal.pone.0010254 (2010). \\
\hline 889 & 36 & Aldon, Y. et al. Rational Design of DNA-Expressed Stabilized Native-Like HIV-1 Envelope Trimers. \\
\hline 890 & & Cell Rep 24, 3324-3338 e3325, doi:10.1016/j.celrep.2018.08.051 (2018). \\
\hline 891 & 37 & Torrents de la Pena, A. et al. Improving the Immunogenicity of Native-like HIV-1 Envelope \\
\hline 892 & & Trimers by Hyperstabilization. Cell Rep 20, 1805-1817, doi:10.1016/j.celrep.2017.07.077 (2017). \\
\hline
\end{tabular}


Han, Y. et al. Monitoring cotranslational protein folding in mammalian cells at codon resolution. Proc Natl Acad Sci U S A 109, 12467-12472, doi:10.1073/pnas.1208138109 (2012).

39 Crotty, S. T Follicular Helper Cell Biology: A Decade of Discovery and Diseases. Immunity 50, 1132-1148, doi:10.1016/j.immuni.2019.04.011 (2019).

40 Mesin, L., Ersching, J. \& Victora, G. D. Germinal Center B Cell Dynamics. Immunity 45, 471-482, doi:10.1016/j.immuni.2016.09.001 (2016).

41 Lu, K. T. et al. Functional and epigenetic studies reveal multistep differentiation and plasticity of in vitro-generated and in vivo-derived follicular T helper cells. Immunity 35, 622-632, doi:10.1016/j.immuni.2011.07.015 (2011).

42 Belshe, R. B. et al. Safety and immunogenicity of influenza A H5 subunit vaccines: effect of vaccine schedule and antigenic variant. J Infect Dis 203, 666-673, doi:10.1093/infdis/jiq093 (2011).

43 Easterhoff, D. et al. HIV vaccine delayed boosting increases Env variable region 2-specific antibody effector functions. JCl Insight 5, doi:10.1172/jci.insight.131437 (2020).

44 McCoy, L. E. et al. Holes in the Glycan Shield of the Native HIV Envelope Are a Target of TrimerElicited Neutralizing Antibodies. Cell Rep 16, 2327-2338, doi:10.1016/j.celrep.2016.07.074 (2016).

45 Nogal, B. et al. Mapping Polyclonal Antibody Responses in Non-human Primates Vaccinated with HIV Env Trimer Subunit Vaccines. Cell Rep 30, 3755-3765 e3757, doi:10.1016/j.celrep.2020.02.061 (2020).

46 Cirelli, K. M. et al. Slow Delivery Immunization Enhances HIV Neutralizing Antibody and Germinal Center Responses via Modulation of Immunodominance. Cell 177, 1153-1171 e1128, doi:10.1016/j.cell.2019.04.012 (2019).

47 Charles, T. P. et al. The C3/465 glycan hole cluster in BG505 HIV-1 envelope is the major neutralizing target involved in preventing mucosal SHIV infection. PLoS Pathog 17, e1009257, doi:10.1371/journal.ppat.1009257 (2021).

48 Ringe, R. P. et al. Closing and Opening Holes in the Glycan Shield of HIV-1 Envelope Glycoprotein SOSIP Trimers Can Redirect the Neutralizing Antibody Response to the Newly Unmasked Epitopes. J Virol 93, doi:10.1128/JVI.01656-18 (2019).

$49 \mathrm{Wu}, \mathrm{X}$. et al. Neutralization escape variants of human immunodeficiency virus type 1 are transmitted from mother to infant. J Virol 80, 835-844, doi:10.1128/JVI.80.2.835-844.2006 (2006).

50 deCamp, A. et al. Global panel of HIV-1 Env reference strains for standardized assessments of vaccine-elicited neutralizing antibodies. J Virol 88, 2489-2507, doi:10.1128/JVI.02853-13 (2014).

51 Steichen, J. M. et al. A generalized HIV vaccine design strategy for priming of broadly neutralizing antibody responses. Science 366, doi:10.1126/science.aax4380 (2019).

52 Zheng, G. X. et al. Massively parallel digital transcriptional profiling of single cells. Nat Commun 8, 14049, doi:10.1038/ncomms14049 (2017).

53 Doores, K. J. et al. Two classes of broadly neutralizing antibodies within a single lineage directed to the high-mannose patch of HIV envelope. J Virol 89, 1105-1118, doi:10.1128/JVI.02905-14 (2015).

$54 \mathrm{Ge}, \mathrm{C}$. et al. Anti-citrullinated protein antibodies cause arthritis by cross-reactivity to joint cartilage. JCl Insight 2, doi:10.1172/jci.insight.93688 (2017).

55 Schorcht, A. et al. Neutralizing Antibody Responses Induced by HIV-1 Envelope Glycoprotein SOSIP Trimers Derived from Elite Neutralizers. J Virol 94, doi:10.1128/JVI.01214-20 (2020).

$56 \mathrm{Xu}$, Z. et al. A DNA-Launched Nanoparticle Vaccine Elicits CD8(+) T-cell Immunity to Promote In Vivo Tumor Control. Cancer Immunol Res, doi:10.1158/2326-6066.CIR-20-0061 (2020). 
57 O'Melia, M. J., Lund, A. W. \& Thomas, S. N. The Biophysics of Lymphatic Transport: Engineering Tools and Immunological Consequences. iScience 22, 28-43, doi:10.1016/j.isci.2019.11.005 (2019).

58 Akbari, O. et al. DNA vaccination: transfection and activation of dendritic cells as key events for immunity. J Exp Med 189, 169-178, doi:10.1084/jem.189.1.169 (1999).

59 Maecker, H. T., Umetsu, D. T., DeKruyff, R. H. \& Levy, S. Cytotoxic T cell responses to DNA vaccination: dependence on antigen presentation via class II MHC. J Immunol 161, 6532-6536 (1998).

60 Morelle, W. \& Michalski, J. C. Analysis of protein glycosylation by mass spectrometry. Nat Protoc 2, 1585-1602, doi:10.1038/nprot.2007.227 (2007).

61 Ledgerwood, J. E. et al. Prime-boost interval matters: a randomized phase 1 study to identify the minimum interval necessary to observe the H5 DNA influenza vaccine priming effect. $J$ Infect Dis 208, 418-422, doi:10.1093/infdis/jit180 (2013).

62 De Rosa, S. C. et al. Robust antibody and cellular responses induced by DNA-only vaccination for HIV. JCI Insight 5, doi:10.1172/jci.insight.137079 (2020).

63 Kanekiyo, M. et al. Mosaic nanoparticle display of diverse influenza virus hemagglutinins elicits broad B cell responses. Nat Immunol 20, 362-372, doi:10.1038/s41590-018-0305-x (2019).

64 Jardine, J. G. et al. HIV-1 broadly neutralizing antibody precursor B cells revealed by germlinetargeting immunogen. Science 351, 1458-1463, doi:10.1126/science.aad9195 (2016).

65 Yang, Y. R. et al. Autologous Antibody Responses to an HIV Envelope Glycan Hole Are Not Easily Broadened in Rabbits. J Virol 94, doi:10.1128/JVI.01861-19 (2020).

66 Khan, K. H. DNA vaccines: roles against diseases. Germs 3, 26-35, doi:10.11599/germs.2013.1034 (2013).

67 Gary, E. N. \& Weiner, D. B. DNA vaccines: prime time is now. Curr Opin Immunol 65, 21-27, doi:10.1016/j.coi.2020.01.006 (2020).

$68 \mathrm{Xu}$, Z. et al. Incorporation of a Novel CD4+ Helper Epitope Identified from Aquifex aeolicus Enhances Humoral Responses Induced by DNA and Protein Vaccinations. iScience 23, 101399, doi:10.1016/j.isci.2020.101399 (2020).

69 Sarzotti-Kelsoe, M. et al. Optimization and validation of the TZM-bl assay for standardized assessments of neutralizing antibodies against HIV-1. J Immunol Methods 409, 131-146, doi:10.1016/j.jim.2013.11.022 (2014).

70 Scheres, S. H. RELION: implementation of a Bayesian approach to cryo-EM structure determination. J Struct Biol 180, 519-530, doi:10.1016/j.jsb.2012.09.006 (2012). 


\section{Figures}

A

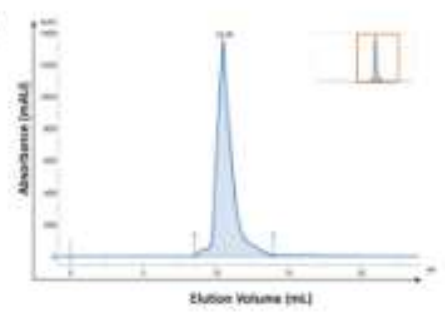

B

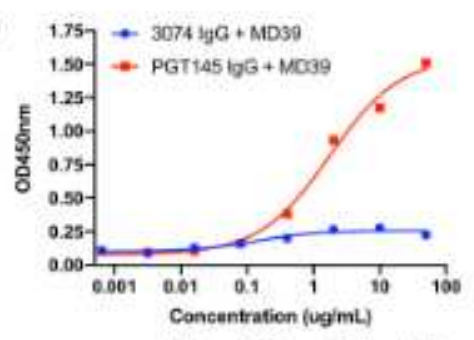

C

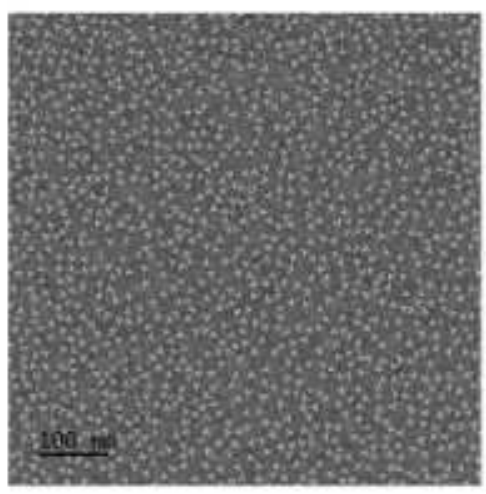

E
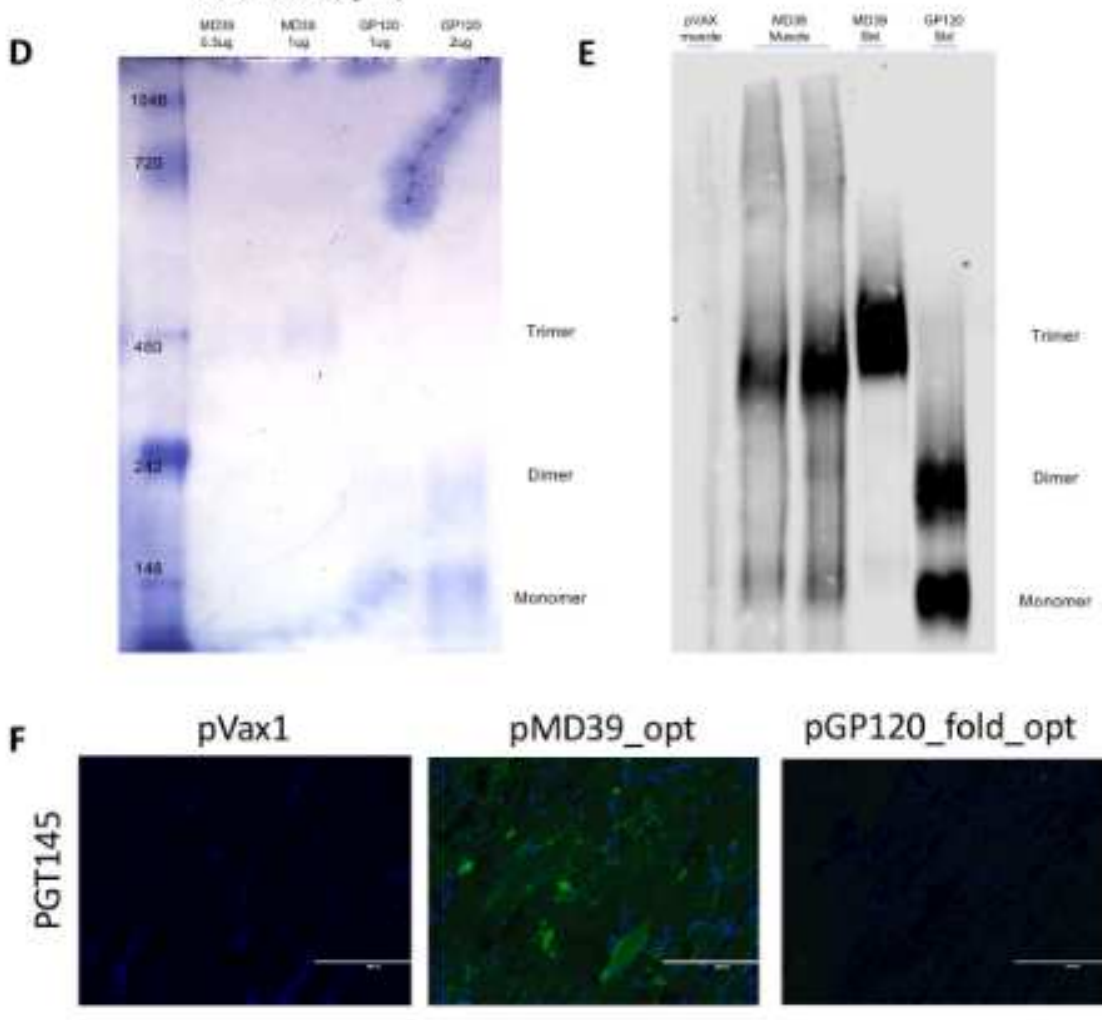

pGP120_fold_opt
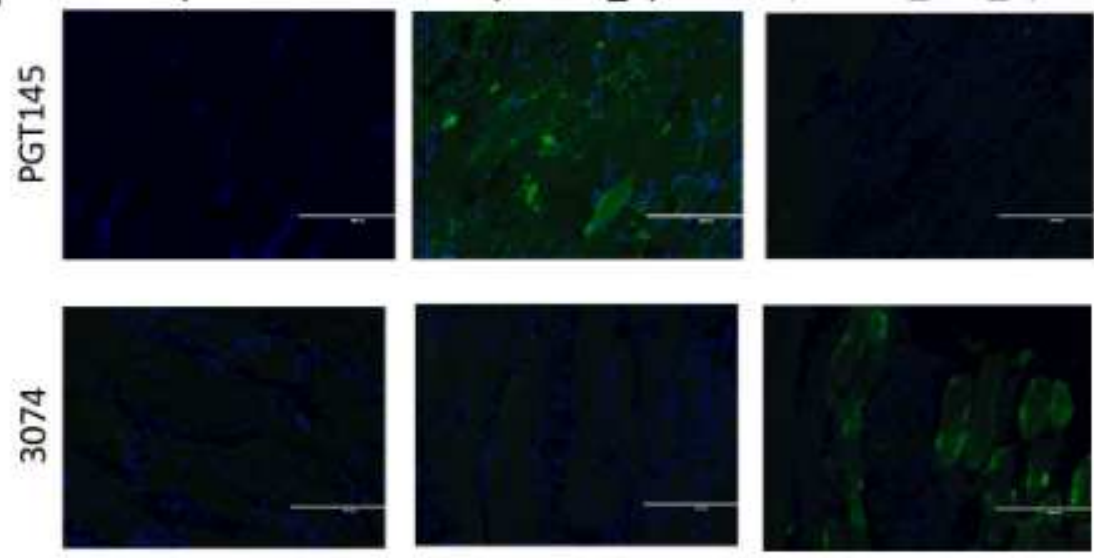

\section{Figure 1}

DNA/EP delivery of BG505.MD39 can enable in vivo expression of conformationally complex NLT. (A) SEC trace of lectin-column purified BG505.MD39 that has been sequence optimized for in vivo expression. (B) Antigenic profile of sequence optimized BG505.MD39 in terms of ELISA binding to V2- 
apex directed bNAb PGT145 and v3-directed non-nAb 3074. (C) nsEM image of SEC purified sequence optimized BG505.MD39. (D) Coomassie staining for the migrations of the purified in vitro expressed BG505.MD39 and BG505 gp120 protein standards on 3-12\% Bis-Tris NATIVE PAGE gels. (E) 2G12-based western analysis to compare the migrations of the in vivo produced BG505.MD39 in muscle homogenates from mice treated with DNA-encoded BG505.MD39 (harvested 4 d.p.i) to those of in vitro produced BG505.MD39 and BG505 gp120 on a 3-12\% Bis-Tris NATIVE PAGE gel. (F) Immunofluorescence analyses of muscle sections from mice treated intramuscularly with DNA-encoded BG505.MD39 or BG505.gp120.foldon (harvested 7 d.p.i) in terms of binding to bNAb PGT145 or non-nAb 3074. 
A

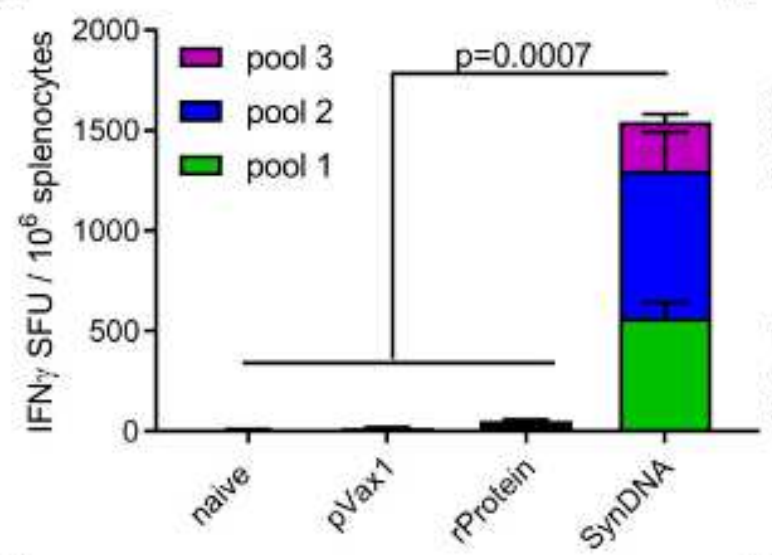

C

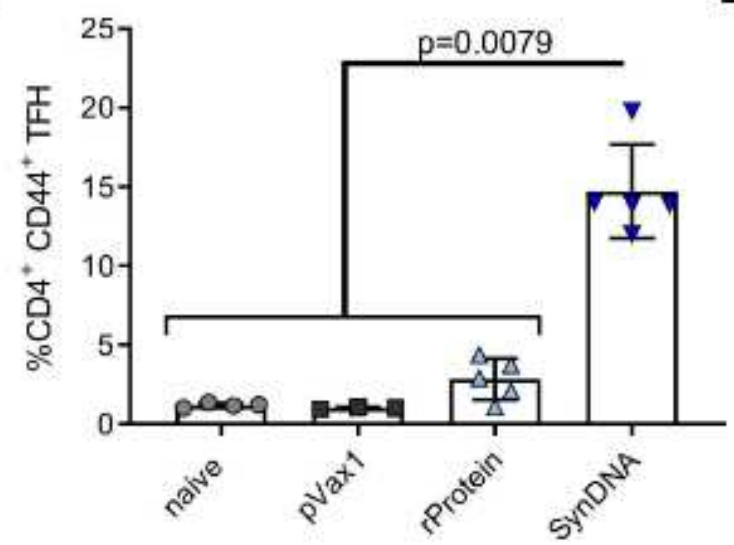

E

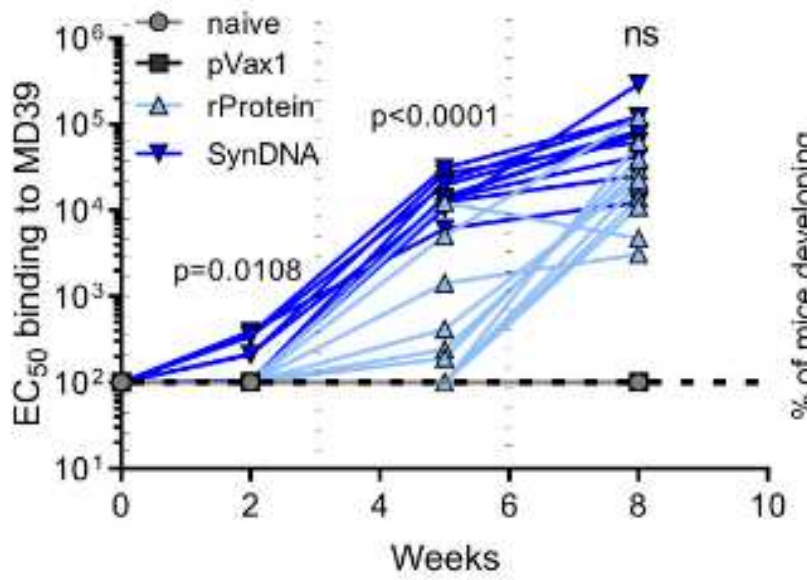

B

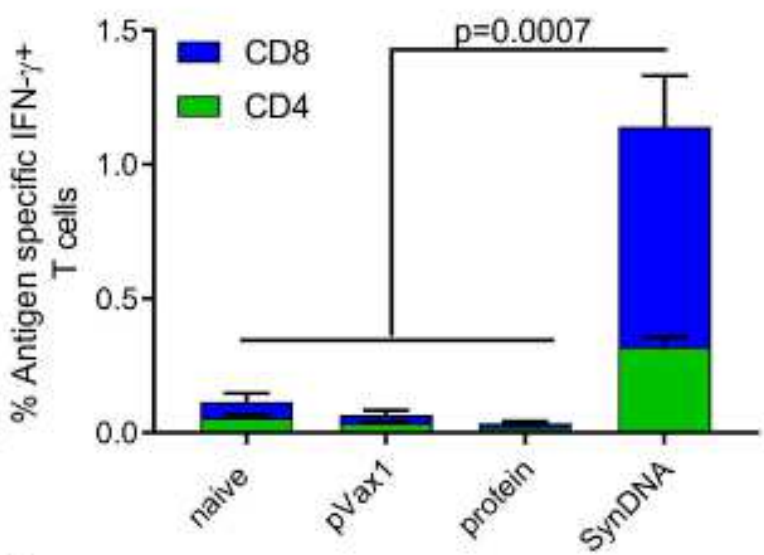

D

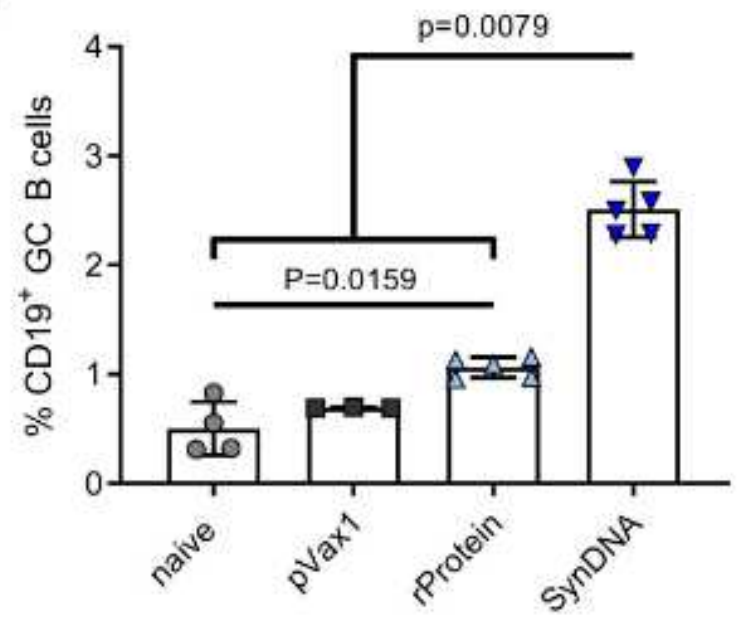

$\mathbf{F}$

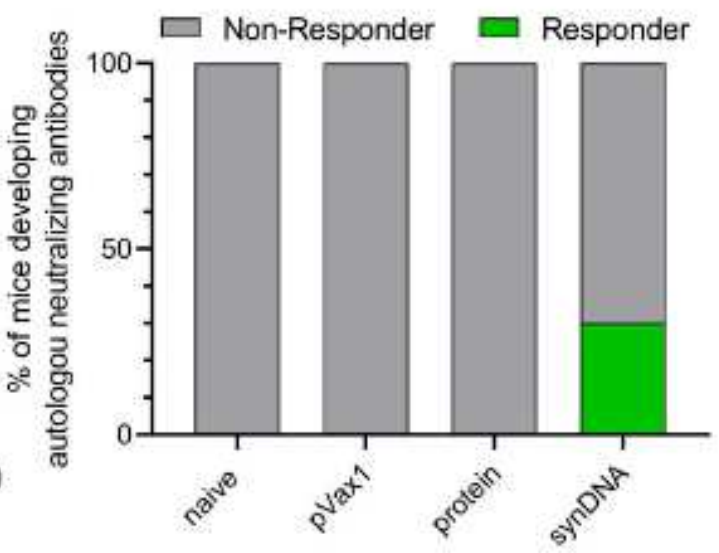

Figure 2

DNA immunization of BG505.MD39 induced stronger T-cell responses and NAb responses than protein immunization in BALB/c mice. All mice in this panel were immunized at Wks 0, 3, 6 with 25ug DNA or 25 ug RIBI-co-formulated protein and euthanized 2 weeks post the final vaccination for cellular analyses. (A and B) Comparison of BG505 Env specific cellular responses in naïve mice, or mice immunized with pVAX plasmid backbone, RIBI-co-formulated protein BG505.MD39, or DNA-encoded BG505.MD39 by IFNy 
ELIspot assay (A) or intracellular cytokine staining (B). (C) Frequencies of CXCR5+PD1+ Tfh cells amongst CD4+CD44+ cells in the draining lymph nodes in naïve mice or 10 days post pVAX1, protein MD39 or DNA BG505.MD39 immunization. (D) Frequencies of GL7+ GC B cells amongst CD19+ B cells in the draining lymph nodes in naïve mice or 10 days post pVAX1, protein BG505.MD39 or DNA BG505.MD39 immunization. (E) Trimer-specific binding antibody responses induced in mice vaccinated with protein or DNA-encoded BG505.MD39 post the first, second and third immunization as determined by ELISA. (F) Frequencies of mice that developed autologous BG505.T332N neutralizing antibodies (ID50 titers greater than 1:45) in naïve mice or mice immunized with PVAX1, protein BG505.MD39 or DNAencoded BG505.MD39 two weeks post the final vaccination. Two independent experiments were performed for each panel in the figure. $\mathrm{N}=10$ mice/group for protein or DNA encoded BG505.MD39, $\mathrm{N}=5$ mice/group for naïve mice or pVAX1 treated mice (A, B, E, F); N=5 mice/group (C-D); each dot or line represents an animal. Error bar represents standard deviation. Two-tailed Mann-Whitney Rank test used to compare groups; 6551 values were adjusted for multiple comparison for panels (A-D); ns: not significant. 
A

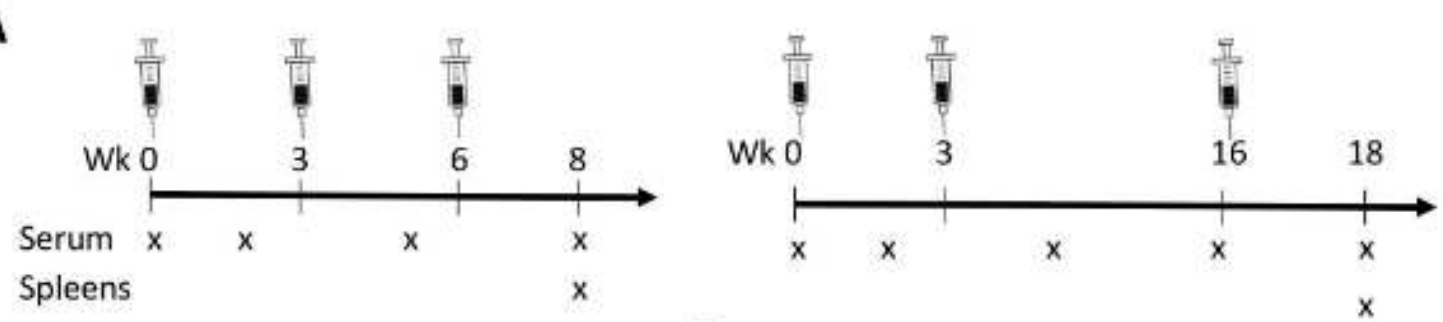

B
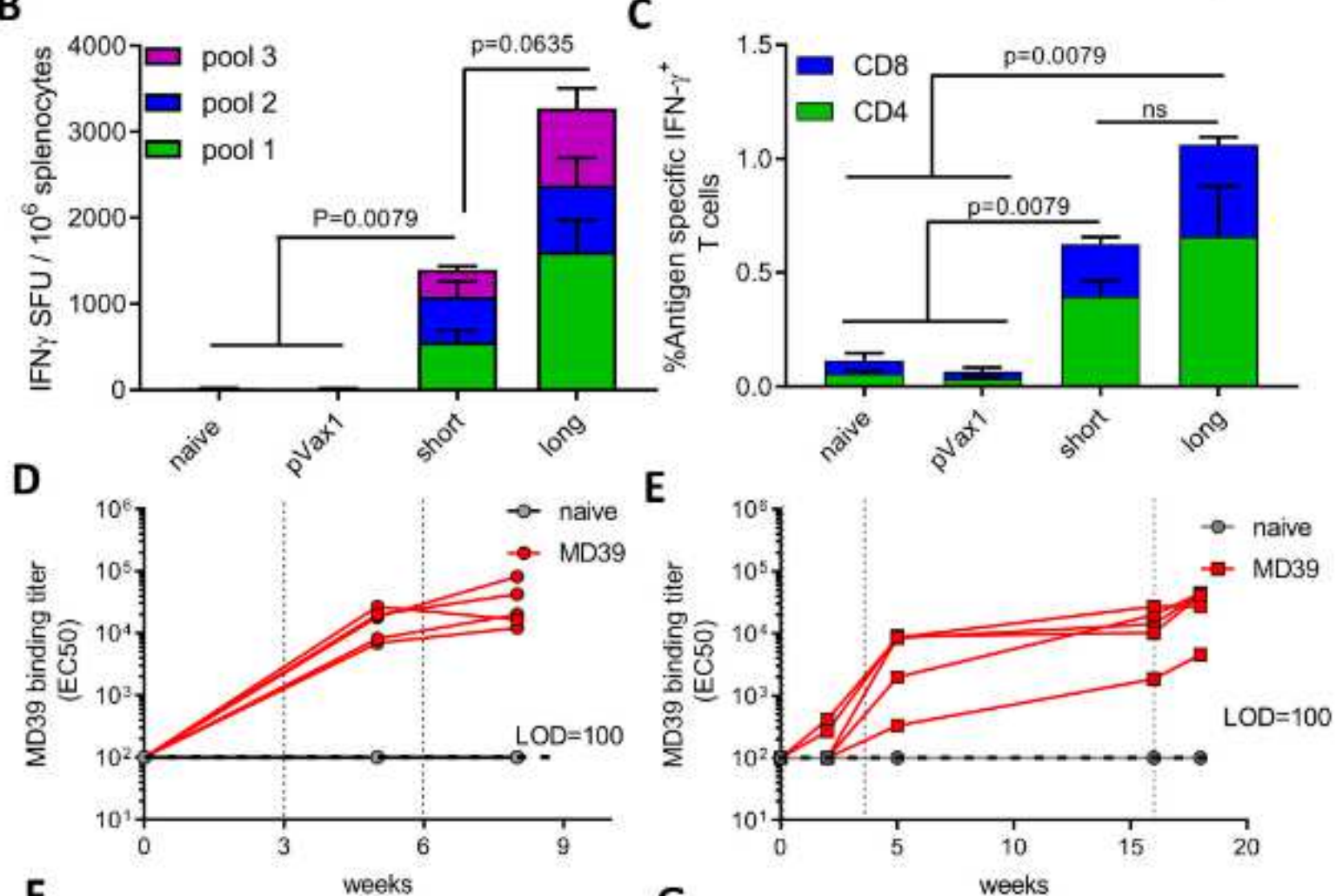

$\mathbf{F}$
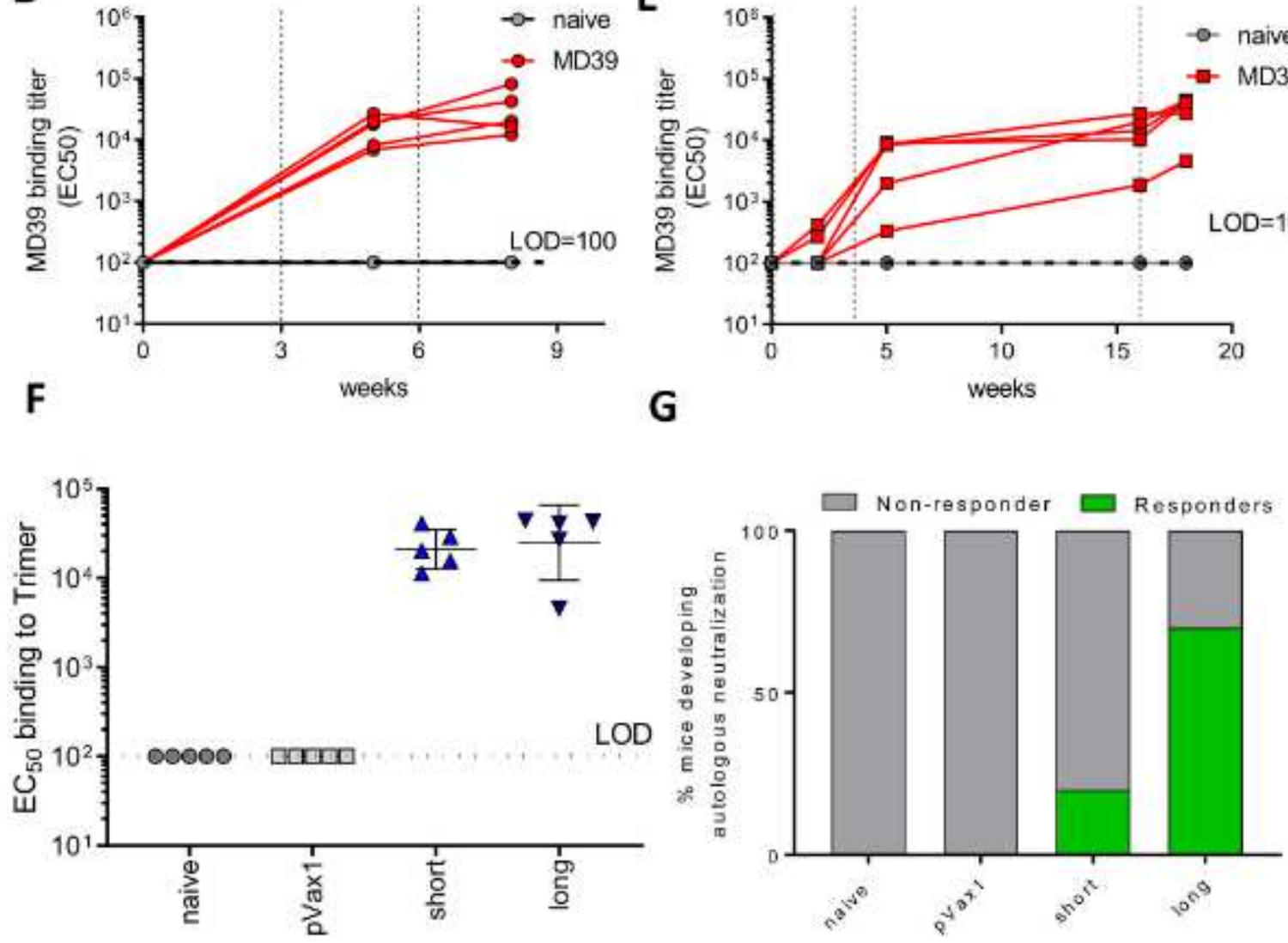

\section{Figure 3}

Prolonging the duration between the second and third immunizations increased frequencies of responders who developed autologous Tier 2 NAbs. Mice in this panel were immunized either with a short scheme (Wks $0,3,6)$ or a long scheme (Wks 0,3 , and 16) with 25ug DNA used at each vaccination. The mice were euthanized 2 weeks post the final vaccination for cellular analyses. (A) Vaccination scheme used in this study. (B and C) Comparison of BG505 Env specific cellular responses in naïve mice, mice 
immunized with pVAX1 plasmid backbone, or those immunized with DNA-encoded BG505.MD39 using either the short or the long scheme by IFNY ELISpot assay (B) or intracellular cytokine staining (C). (D and E) Time course of trimer-specific binding antibodies in naïve mice or mice vaccinated with DNA-encoded BG505.MD39 using either the short scheme (D) or the long scheme (E) as determined by ELISA. (F) Comparison of trimer specific binding antibodies at the final timepoint (two weeks post the third vaccination) in mice immunized with DNA-encoded BG505.MD39 using either the short or the long scheme. (G) Frequencies of mice that developed autologous BG505.T332N neutralizing antibodies (ID50 titers greater than 1:45) in naïve mice, mice immunized with pVAX1, or mice immunized with DNA665 encoded BG505.MD39 using either the short or the long scheme two weeks post the final vaccination. Two independent experiments were performed for each panel in the figure. $N=5$ mice/group for $(A-F)$; $\mathrm{N}=10$ mice/group (G); each dot or line represents an animal. Error bar represents standard deviation. Two tailed Mann-Whitney Rank test used to compare groups; $\mathrm{p}$-values were adjusted for multiple comparison for panels (B-C); ns: not significant. 
A

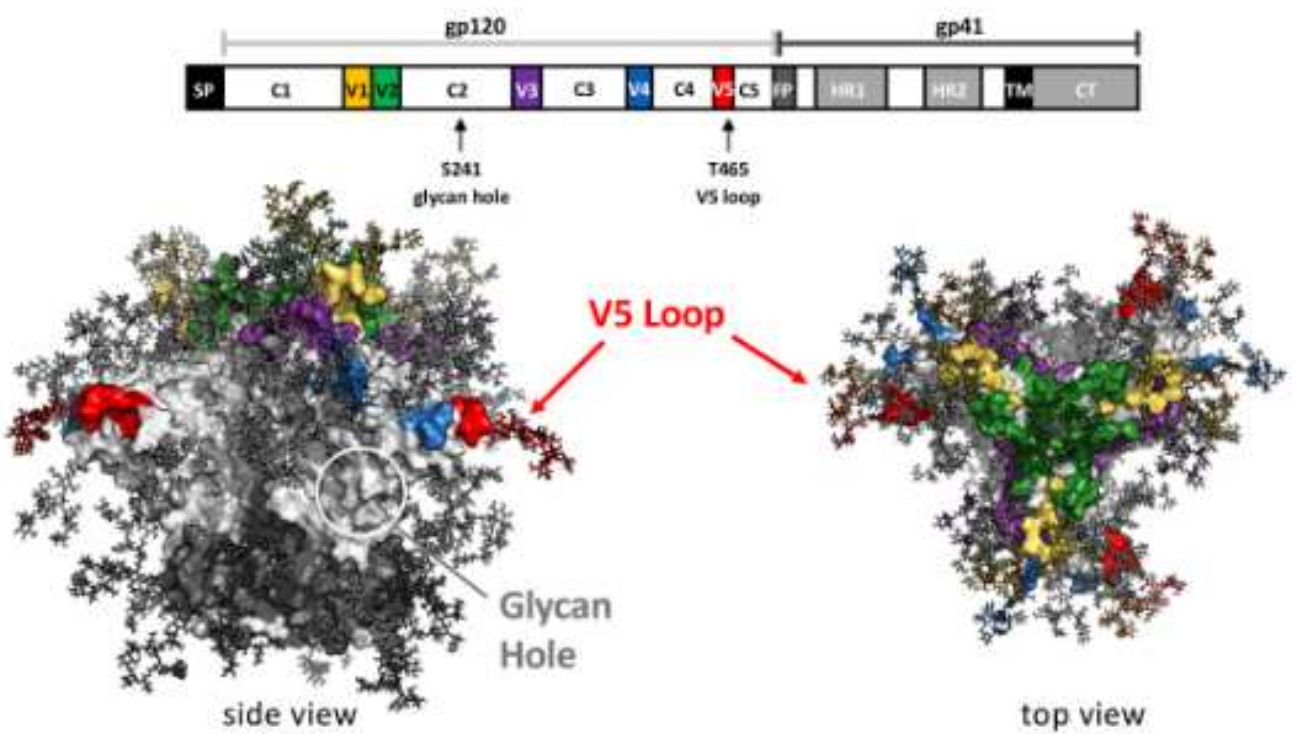

B
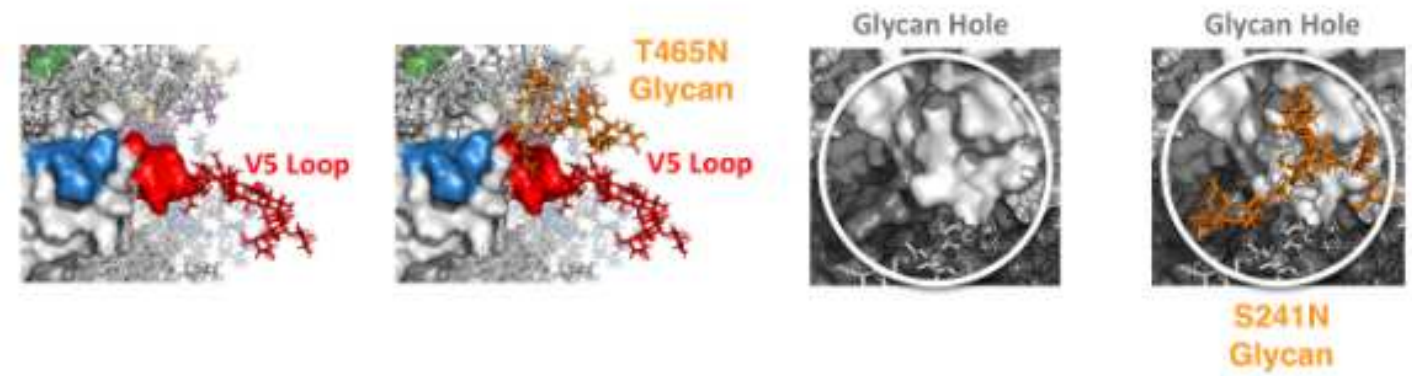

C

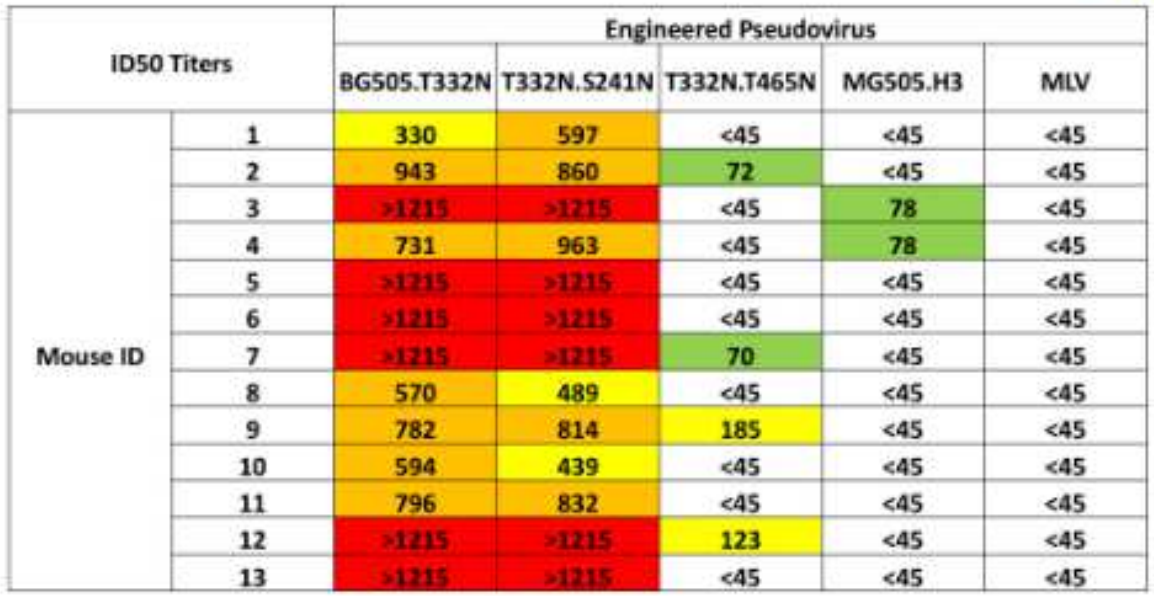

D

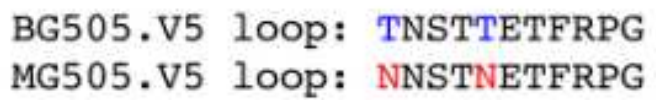

Figure 4

Screening with engineered BG505.T332N pseudo-viruses with modified glycosylation sites mapped induced murine NAb to the V5-loop of Envelope. Mice in this panel were immunized with variations of DNA-encoded BG505.MD39 at Wks 0, 3 and 16 with 25ug DNA used at each vaccination and identified to possess potent BG505.T332N neutralizing activities 2 weeks post the final vaccination. (A and B) Rosetta modeling to demonstrate the locations of the engineered glycosylation sites (A) and the V5 loop (B) on 
the NLT. (C) Neutralization ID50 titers of heat-inactivated mice sera against listed pseudovirus isolates. (D) Sequence alignment of the V5 loops for BG505.W6M.ENV.C2 and MG505.WOM.ENV.H3.

A

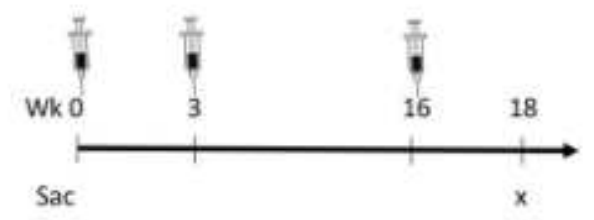

B

C
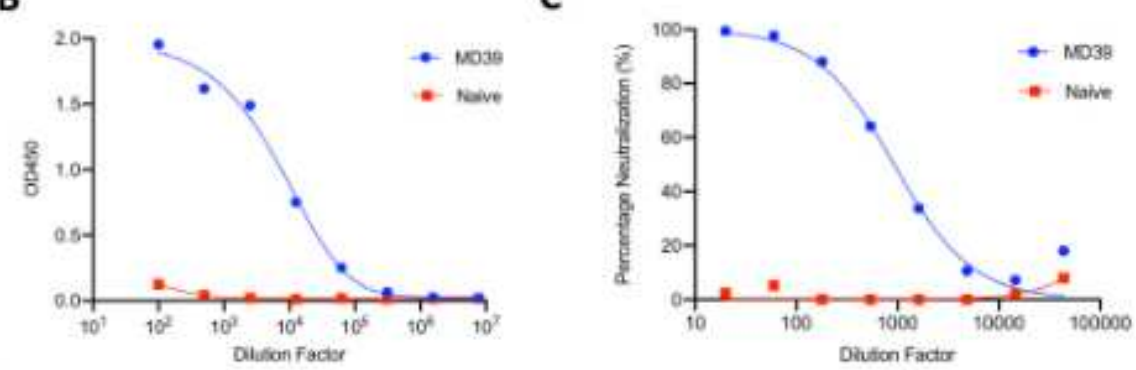

D
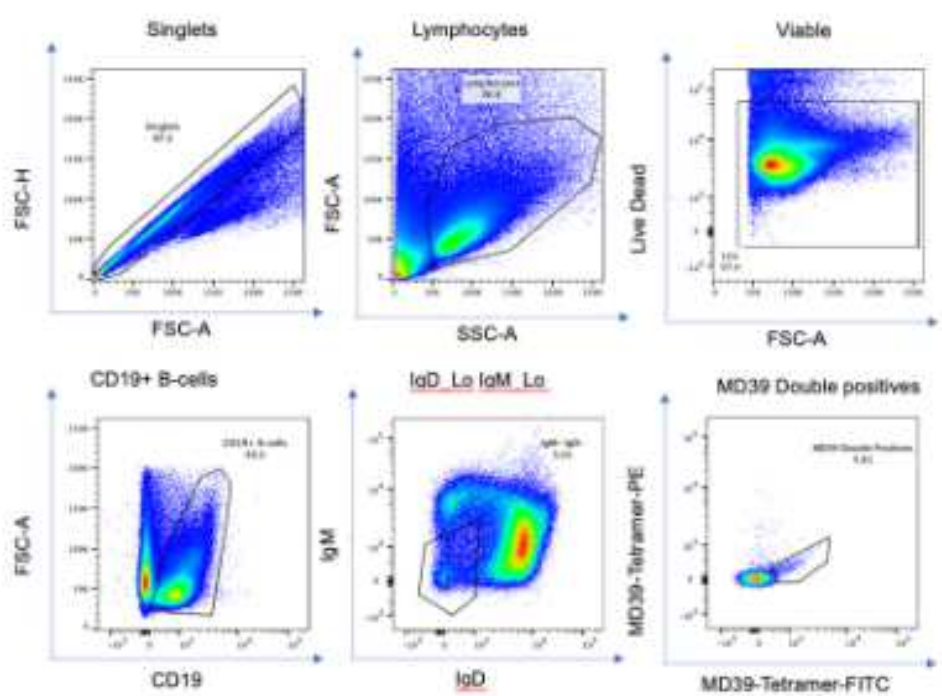

E

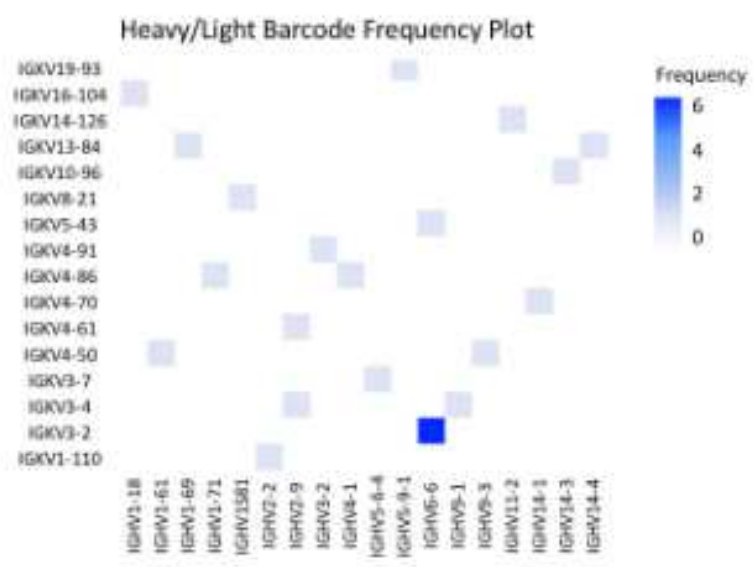

Figure 5

Isolation of trimer specific monoclonal antibodies from an animal (272.8) with potent neutralization activity against BG505.T332N post vaccinations with DNA-encoded BG505.MD39. (A) Vaccination and tissue collection scheme for 272.8. (B) Trimer-specific binding antibody responses in 272.8 two weeks 
post the final vaccination. (C) Autologous BG505.T332N neutralizing activity induced in 272.8 two weeks post the final vaccination. (D) Gating strategy used to isolate antigen-specific CD19+ IgD IgMBG505.MD39-Tetramer-FITC+ BG505.MD39-Tetramer-PE+ B-cells from spleen and lymph nodes of 272.8 two weeks post the final vaccination. (E) Characteristics of isolated murine monoclonal antibodies in terms of germline $\mathrm{VH}$ and $\mathrm{VL}$ gene usage.

A

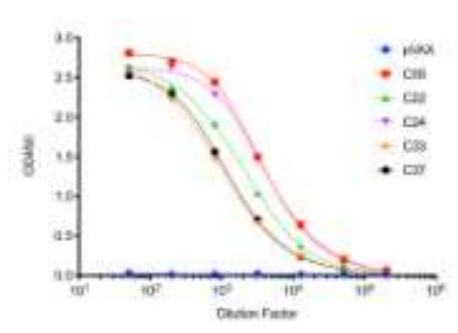

D

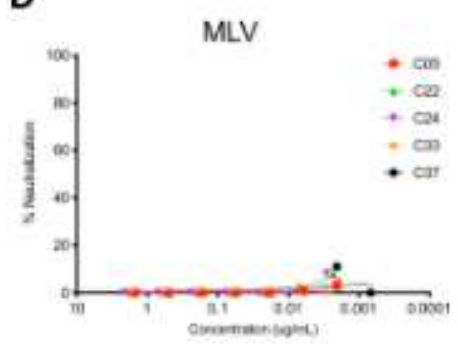

B

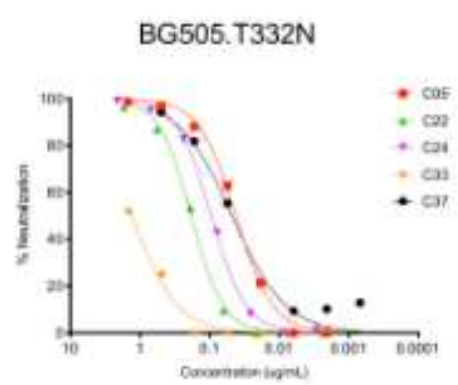

E
C

\section{BG505.T332N.T465N}

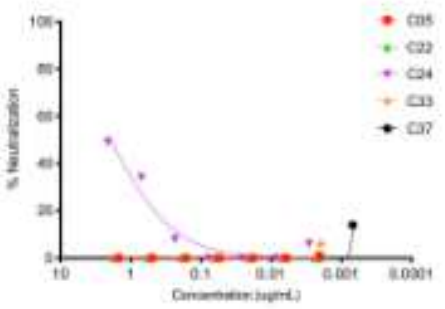

\begin{tabular}{|c|c|c|c|c|c|}
\hline IC50 (ug/mL) & C05 & C22 & C24 & C33 & C37 \\
\hline BG505.T332N & 0.039 & 0.174 & 0.095 & 1.332 & 0.047 \\
\hline BG505.T332N.T465N & $>50$ & $>50$ & 2.1 & $>50$ & $>50$ \\
\hline MLV & $>50$ & $>50$ & $>50$ & $>50$ & $>50$ \\
\hline
\end{tabular}

F

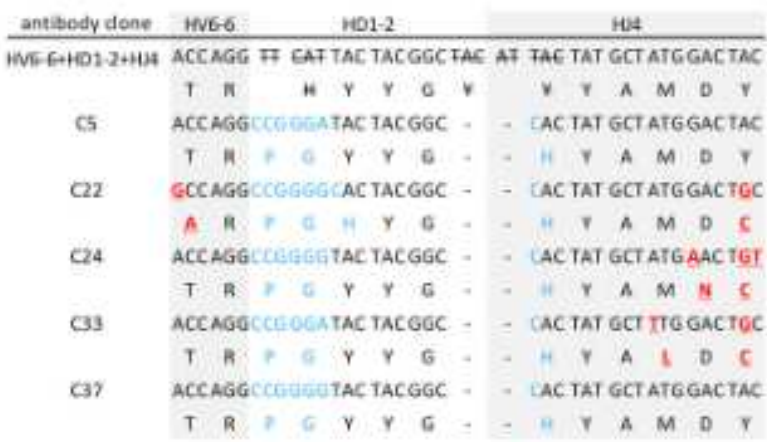

\begin{tabular}{|c|c|c|c|c|}
\hline antibody dene & & $\mathrm{xV}_{3} \cdot 2$ & & $\mathrm{X} / 2$ \\
\hline \multirow[t]{2}{*}{$\mathrm{k}_{3} 32+\mathrm{k12}$} & CAGCMAGT & TAACGAGGT CCF & cE & FG TAC ACG \\
\hline & Q Q 5 & $x \in V$ & & $r t$ \\
\hline \multirow[t]{2}{*}{ C5 } & CAGCAAAG: & AAGGAGGT CC & - & - tatacG \\
\hline & a $\quad$ a 5 & K $\mathrm{i} \quad \mathrm{V} F$ & 4 & $=Y T$ \\
\hline \multirow[t]{2}{*}{$\mathrm{C} 22$} & CAGCAAAGT & AAGGAGGT CC & $=$ & - TACACG \\
\hline & a $\quad$ a 5 & $x \quad \mathrm{f} \quad \geqslant$ & - & $=r T$ \\
\hline \multirow[t]{2}{*}{ Q24. } & CAGCAAAGT. & AAGGAGGT CCE & . & - TACACG \\
\hline & a $a 5$ & $X \quad \mathrm{E} \quad \mathrm{V} P$ & - & $=X T$ \\
\hline \multirow[t]{2}{*}{$\mathrm{CB}$} & CAOCAAMOT & AAGSAG OTI CCE & - & $=T A C A C O$ \\
\hline & a a s & $x \notin \mathrm{V} F$ & - & $-Y T$ \\
\hline \multirow[t]{2}{*}{$\mathrm{Car}$} & CAGCAAAGT. & AAGGAGGTICCL & - & - TACACG \\
\hline & a $\quad$ Q 5 & $X \quad \mathrm{f} \quad \mathrm{V}$ & - & $=Y T$ \\
\hline
\end{tabular}

G
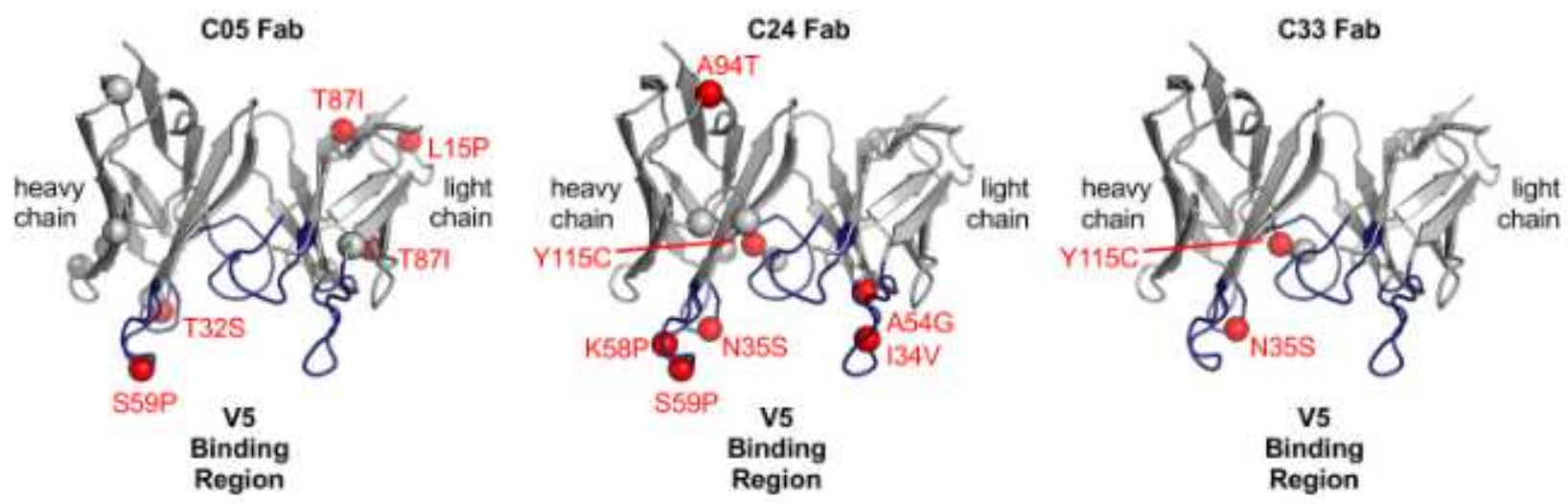

Figure 6 
Isolated trimer-specific murine MAbs neutralized BG505.T332N pseudovirus in a V5- dependent fashion. (A) Trimer specific binding of 5 isolated murine MAbs and PGT128 in Expi293F transfection supernatant. (B - D) Neutralization of BG505.T332N (B), BG505.T332N.T465N (C) and MLV (D) pseudo-viruses with varying concentrations of murine trimer-specific MAbs or PGT128. (E) Determined IC50 values in ug/mL of trimer-specific murine MAbs for BG505.T332N, BG505.T332N.T465N or MLV. F. Amino acid alignments of the HCDR3 and LCDR3 regions of the identified BG505.MD39-specific murine antibody clones as compared to the corresponding germline sequences. G. Predicted Rosetta structures of the Fabs of clones C05, C24 and C33, positions mutated from the germline sequences are displayed in red. 
A

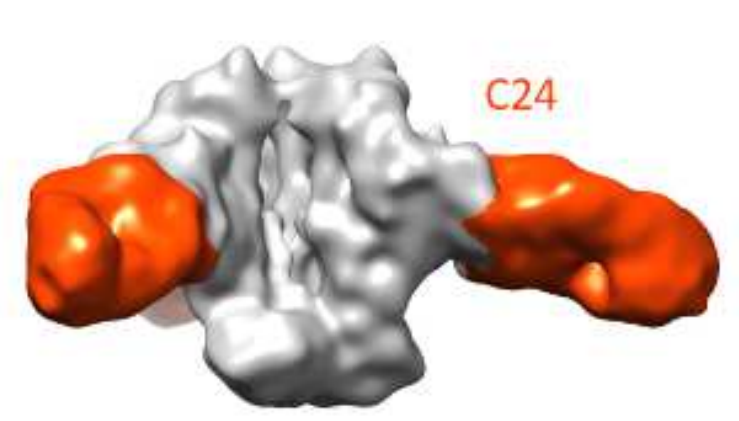

B

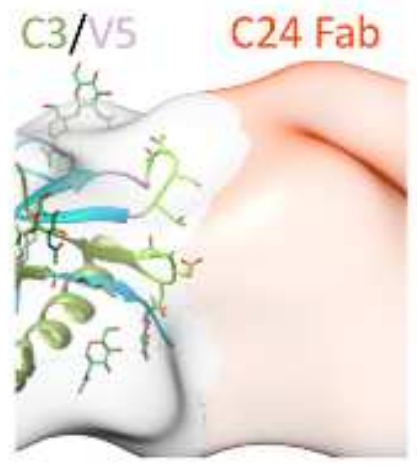

C
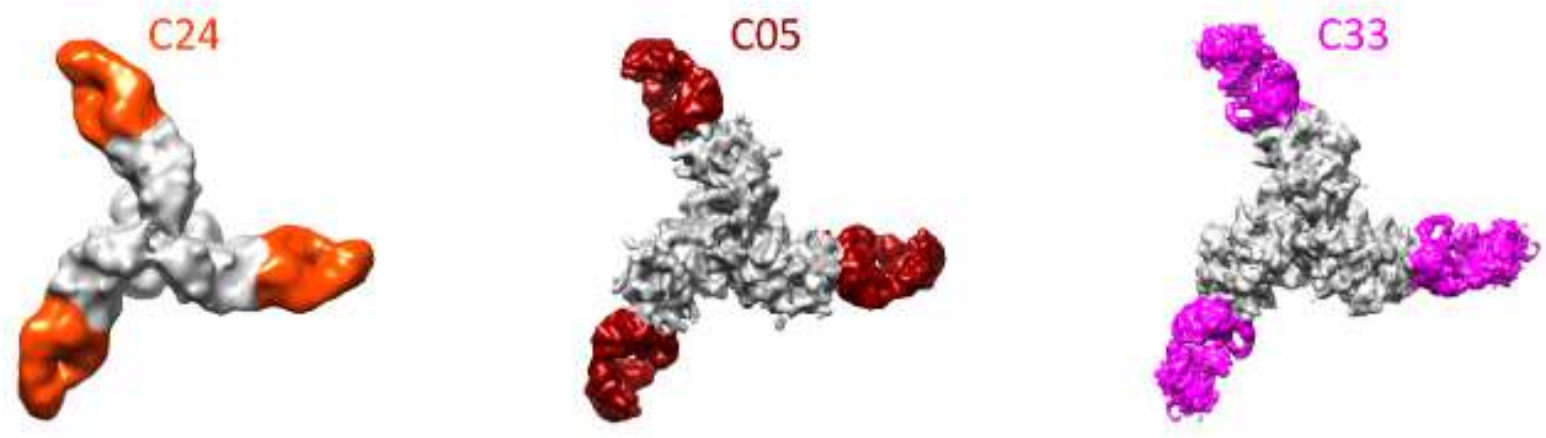

D
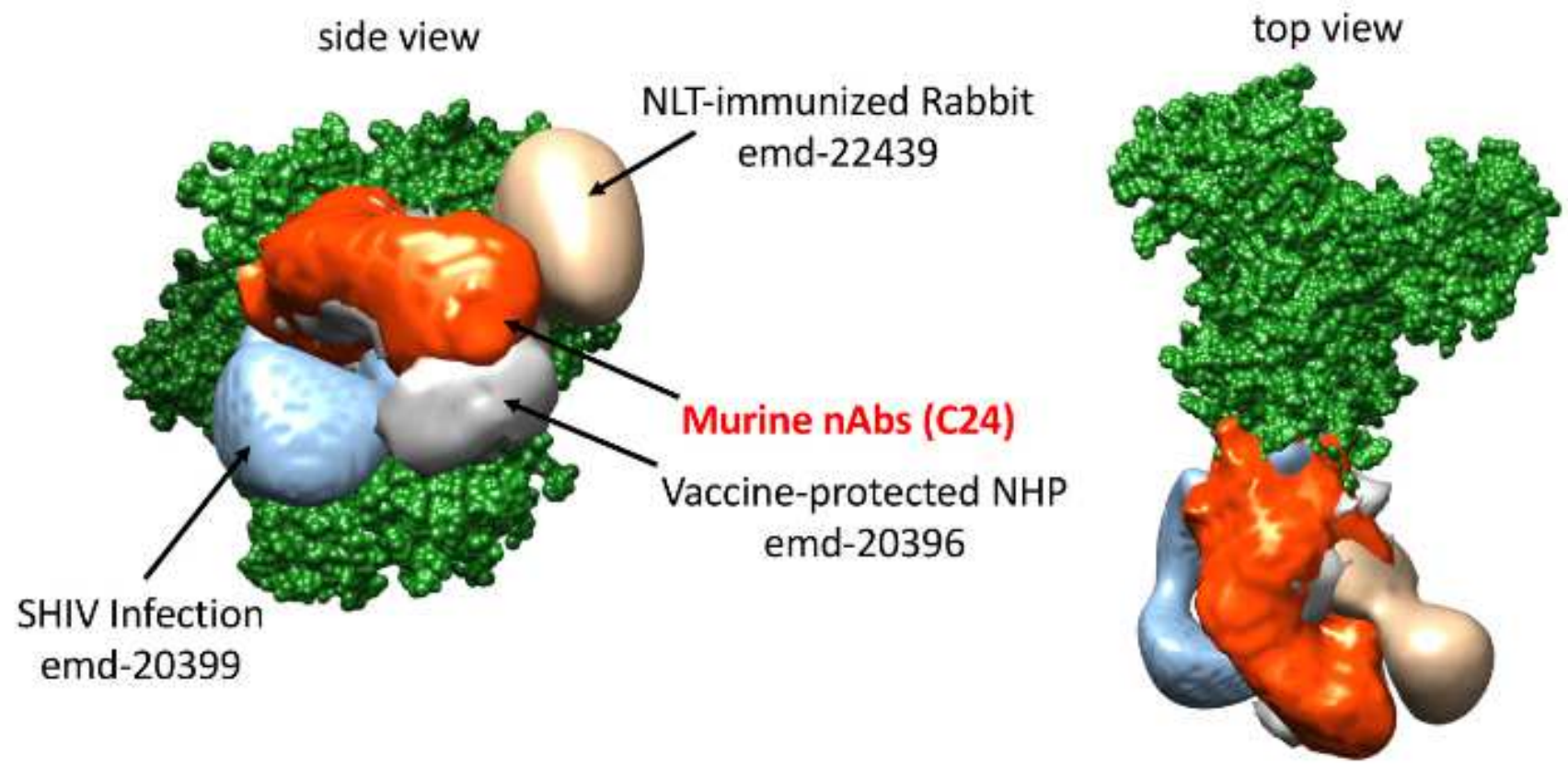

Figure 7

Cryo-EM based structural characterization of Fabs of murine nAbs bound to BG505.MD39. (A) Cryo-EM structure of BG505.MD39 in complex with murine neutralizing Fab C24 (BG505.MD39 in grey surface and C24 Fab in orange surface). (B) Interface of structure in (A) with Env gp120 epitope shown as cartoons and lines. (C) Top view of three cryo-EM structures: C24 (orange), C05 (red) and C33 (magenta) (D) Structural alignment using density of complexed Env for four C3/V5 targeting abs from a SHIV infected 
NHP (blue), a NLT-immunized rabbit (white), a NLT-vaccinated and challenged NHP (grey) and murine nAbs C24 (orange). Both side and top view are shown and Env is shown in green.

\section{Supplementary Files}

This is a list of supplementary files associated with this preprint. Click to download.

- MD39paperfigure20200903v10Supplemantary.pdf

- Rawdatatrimermanuscript.xlsx

- Table1.png 